\title{
Objective Analysis for Numerical Weather Prediction
}

\author{
By A. Hollingsworth \\ ECMWF, Shinfield Park, Reading, U.K. \\ (Maeuscript received 22 November 1986, in revised form 20 April 1987)
}

\begin{abstract}
The field of objective analysis for numerical weather prediction is enjoying rapid development because of theoretical advances in analysis methods coupled with progress in observing and computing technology. This review considers current and projected work on objective analysis under three main headings: theory, verification and practice.

Recent developments in the theory suggest that there are close links between all analysis methods based on optimality principles. Moreover, simpler analysis methods such as successive correction can be modified to converge to an optimal method in simple circumstances. The basic job of any analysis algorithm is to filter random observation error from the data and then interpolate the filtered data to a regular grid. A new viewpoint on Optimum Interpolation shows the algorithm in a simple light with an intimate connection between the filtering and interpolation capabilites.

Verifications of observations, forecasts, and analyses are reviewed, using statistics on one-point and two-point height and velocity correlations. The two-point correlations are inter-related through the kinematic equations of two-dimensional turbulence. In the limit of vanishing spatial lag they degenrate to the one-point correlations. These diagnostics offer a means to compare the error characteristics of different observing systems. When used to verify short-range forecasts, they provide a complete description of the structure of the forecast errors. Phillips' simple theory of forecast errors can reproduce many of the features of the empirically determined structures. New results indicate that there may be a need for anisotropic and non-separable correlation functions for analysis.

The correlation diagnostics offer new and rigorous ways to estimate analysis error at observation points. They provide a quick and easy measure of the efficiency of an analysis system. They should prove useful in identifying the reasons for system dependence in the results of observing system experiments.

In discussing current and future research we consider the special problems of analysis in data rich areas over land and near mountains; the problems of analysis over the midlatitude oceans; and then the problems of tropical analysis. Extensions of $\mathrm{O} / \mathrm{I}$ offer new approaches to these problems which will be explored in the near future. In the slightly longer term, new developments in the theory of 4-dimensional analysis may well supersede current methods. Finally we consider developments in data quality control, an area of great importance for all other developments. New developments here, using Bayesian methods, offer promise.
\end{abstract}

\section{Introduction}

In recent years we have seen the advent of successful global operational forecasting (Bengtsson, 1985); we have also seen the successful production of the FGGE III-b data, providing global analyses for 1979 from a vast and heterogeneous observational database. The development of objective analysis and data assimilation methods up to the completion of the main FGGE analyses is discussed in the review by Bourke, Seaman and Puri (1985). Their discussion pays particular attention to the many simulation studies that 
were undertaken to prepare the observational plan for FGGE. Objective analysis of the FGGE data has been the subject of a number of extensive reviews (National Research Council, 1985 ; ECMWF, 1984).

There has been notable progress in understanding the kinematics, statistics, and mathematics of meteorological analysis methods in recent years. A theoretical framework is available which connects the many apparently unrelated methods which have been developed for meteorological analysis. The basic starting point in all methods is that the observations are insufficient to determine the continuous (or discrete) fields of interest. One must use additional $a$-priori information to resolve the under-determined problem. The $a$-priori information may be a forecast and the statistics of its errors, or constraints of continuity and/or differentiability on the solutions. The $a$-priori information and the observational information are combined in a Bayesian (maximum likelihood) or minimumvariance calculation which minimises a functional of the unknown solution; the advantage of the Bayesian methods is that they can handle non-gaussian statistics.

The method of optimal interpolation $(\mathrm{O} / \mathrm{I}$; Gandin, 1963; Eliassen, 1954) has an important role in establishing links between the many different analysis methods in existence. (The method is also called statistical interpolation since the optimum statistics can never be known exactly). The multivariate theory of $\mathrm{O} / \mathrm{I}$ has been further developed (Hollingsworth and Lönnberg, 1986; Lönnberg and Hollingsworth, 1986, hereafter called HL/LH) using the kinematics of two-dimensional turbulence. These kinematic methods extend the capabilities of the $\mathrm{O} / \mathrm{I}$ formalism in large scale, synoptic scale and meso-scale analysis and in tropical analysis. In addition they provide poweful diagnostic tools.

Assimilation systems have improved to the point where the 6-hour forecast is as accurate as the observations in data-rich areas of the Northern Hemisphere (Hollingsworth et al., 1986). This offers new possibilities for improving the WWW data through long term monitoring. It also places new demands on that data since the noise to signal ratio is now approaching 1 , if both noise and signal are measured relative to the first-guess. Improved communication between data producers and data users would be useful as the users know a lot about errors in the data.

The utilisation of available data over the oceans is still far from satisfactory. In the $\mathrm{O} / \mathrm{I}$ context there is a need for more extensive documentation of forecast error statistics over the ocean. Further efforts are also needed to simulate the oceanic data distributions over land so that one may use independent data to define the three dimensional structure of the oceanic forecast errors. The extensive operational experiment planned by WMO for $1987 / 88$ in the North Atlantic will be a particular stimulus to such efforts.

Analysis methods in the Tropics have improved through the imposition of physical balance requirements (Krishnamurti et al., 1983, 1984) and the recognition of the importance of treating the very largest scales in the analysis (Cats and Wergen, 1982 ; Daley Cats and Wergen, 1986); further gains may come from enhancements in the synoptic-scale wind analysis to take account of divergence (Daley, 1985). In conjunction with refinements in model resolution, parameterisation and orography, the analysis changes that have been implemented so far have led to marked improvements in the quality of operational forecasts in the Tropics. Despite these improvements, the over-riding problem remains the lack of observations in the Tropics.

To maximise the utilisation of the available observations, more effective methods will be needed to incorporate the time dimension in the analysis algorithm. The adjoint method proposed by Le Dimet and Talagrand (1986) appears to offer a sound mathematical foundation for tackling this problem. As pointed out by Lorenc (1986), the more explicit use of an accurate model in the assimilation also offers the possibility of including in the analysis non-linear aspects of balance, such as strong fronts in balance with a large-scale front-genetic field.

The plan of the paper is as follows. We begin in section 2 by reviewing the structure 
of current assimilation systems, emphasising the need to use the most accurate model possible in the data assimilation; this has only recently been achieved at some centres. We also discuss the need for accuracy in the multivariate interpolations of the background field from the model grid to the observation position.

Theoretical work establishing connections between many different analysis methods is discussed in section 3, together with experimental work on comparisons of the accuracy of analysis methods.

The basic task of any analysis system is to filter as much error as possible from the observations and then to make an interpolation of the resulting information to a regular grid. The intimate connections between the filtering capabilities and the interpolation capabilities of $\mathrm{O} / \mathrm{I}$ are discussed in section 4.

Section 5 review recent developments using the kinematics of two-point velocity and geopotential correlations, and discusses how the capabilities of observing systems may be evaluated by comparison of atmospheric spectra observed by the different systems. Section 6 discusses progress in the determination of the correlation structure of forecast errors. Theoretical explanations of the empirically determined structures are also considered. One-point velocity correlations are used to survey the anisotropies in the forecast errors. Section 7 reviews the many different methods which have been used to estimate analysis error. Particular attention is given to new methods which permit a proof that all useful information has been extracted from the observations.

Section 8 considers current and future research in operational objective analysis including the problems of high resolution analysis in data-rich land areas and in the presence of mountains, the problems of analysis over the mid-latitude oceans, the problems of analysis in the Tropics, and new developments in quality control. The main themes of the paper are summarised in the concluding section.

Inevitably, there are modest overlaps with the companion reviews by Machenhauer (1987), and Puri and Gauntlett (1987), but we have tried as far as possible to avoid duplication. Inevitably also, most attention is given to the work of present and former colleagues. Our viewpoint has been described as EC-centric, but it is hoped that it is nonetheless useful.

\section{The structure of current assimilation systems}

The basic structure of most current assimilation systems is similar. A forecast model generates a 6 -hour or 12 -hour forecast to provide a background field for the next analysis; the background field is interpolated to the observation position; the differences between observation and first-guess are filtered, interpolated spatially, and added to the background field to form the analysis, and the process is repeated.

Current operational analysis systems at the larger centres use one of two forms of 4dimensional assimilation, either a continuous insertion or an intermittent insertion (Bengtsson, 1975; Miyakoda, 1985; Lorenc, 1981; Bergman, 1979; McPherson et al., 1979; Kistler and Parrish, 1982 ; Dey and Morone, 1985 ; Baker 1982). In both of these approaches the methods of Non-Linear Normal Mode Initialisation (NNMI, Machenhauer, 1977; Baer, 1977; Temperton and Williamson, 1980; Williamson and Temperton, 1980) can play an important role. In the intermittent insertion method used at most of the major Centres (Gustafsson, 1981) NNMI plays a vital role in assuring the balance of the background field which is essential in quality control (Van Maanen pers. comm., 1982) and for the validity of the basic multivariate assumptions (Lorenc, 1981, 1986). One goal of research on intermittent assimilation has been the unification of the analysis problem and the assimilation problem (Daley, 1978; Phillips, 1981b ; Tribbia, 1982 ; Williamson and Daley, 1983). Variational formulations of the problem are amenable to solution by direct methods and by adjoint methods. In continuous assimilation schemes (Bengtsson, 1985; Lyne, 1982 ; Miyakoda, 1985; Miyakoda et al., 1986) the differences between observation and background may be inserted in the model in a repetition of the last forecast, and 
in the forecast which generates the background for the next analysis.

Success in data assimilation requires accuracy in all four phases of the calculation: the forecast (para 2.2), the forward interpolation, (para 2.3), the analysis (section 3 et seq.) and the initialisation (para 2.4). There have been substantial improvements in most of these areas in recent years. To set the scene for these discussions, it is useful to review recent progress at one institute chosen as an example.

\subsection{Impact of model and analysis changes on forecast skill at ECMWF}

To get an overall impression of the impact of analysis improvements and model improvements we consider the ECMWF forecast performance for a four year period, June 1982 to April 1986. Fig. 1 (from Shaw et al., 1987) shows the rms $500 \mathrm{mb}$ geopotential forecast errors for the eleven months from June through April in the four successive years 1982/83-1985/86, for each hemisphere between latitudes $20^{\circ}$ and $80^{\circ}$. The May data were excluded because some of the more important model and analysis changes discussed below occurred in May.

The 1982/83 forecasts, for the first of these periods, were made with the N48 grid point model (Burridge and. Haseler, 1977; Tiedtke et al., 1979; Hollingsworth et al., 1980; Bengtsson and Simmons, 1983). Between the first and second period there was an important model change. The forecasts for the second period, 1983/84, were made with a T63 spectral model using the same physical parameterisation package. This spectral model had been shown to have superior forecast performance (Girard and Jarraud, 1982; Simmons and Jarraud, 1984) and replaced the grid-point model as the operational model in late April 1983. At the same time the orographic representation was modified to an envelope orography (Wallace et al., 1983).

Between the second and third periods there was a major change in analysis and some changes in the model. The changes in the analysis system in May 1984 (Shaw et al., 1984, 1987) affected many aspects of the system including quality control, data selection algorithms, and structure functions. In addition an important change was made to the vertical interpolation scheme in Nov. 1984 (Unden, 1985). The forecasts for the third period, $1984 / 85$, were made with the same model as in the $83 / 84$ period except for two changes in the radiation scheme. The first change, in May 1984, was the introduction of a diurnal cycle (Tiedtke and Slingo, 1984). The second change, in December 1984, modified the representation of the transmission functions and led to a substantial reduction of the forecast error biases in the tropical temperatures in the medium range (Ritter 1985).

Between the third and fourth periods there were major changes in the model. In May 1985 the resolution of the spectral model was upgraded to T106 (Jarraud, Simmons and Kanamitsu 1985). At the same time there were major changes to the parameterisation package. A new representation of shallow convection was introduced, the partitioning of moisture convergence between heating and moistening in the convection scheme was changed substantially, and the cloud parameterisation was radically revised (Tiedtke et al. 1987).

There were clear improvements at all forecast ranges in the Southern Hemisphere (Fig. 1a) between the first and second periods, after the introduction of the T63 spectral model in May 1983, and again in 1984/85 after the introduction of the analysis modifications in May 1984. There were further gains resulting from the introduction of the T106 model with improved physics in 1985; these improvements were somewhat larger than expected from increases in resolution alone (Jarraud, Simmonsand Kanamitsu, 1985, Tiedtke et al. 1987) and may be partly attributable to the physics changes and partly to the gradual build-up in the number of drifting buoys for TOGA from late 1985 onward. Over the four year period the largest improvement in the Southern Hemisphere forecasts clearly came from the analysis improvements.

In the Northern Hemisphere, Fig. 1b, the results are quite different. The introduction 
Southern Hemisphere 500mb RMS Errors 1982/3 1983/4 1984/5 1985/6 Jun-Apr (11mo)

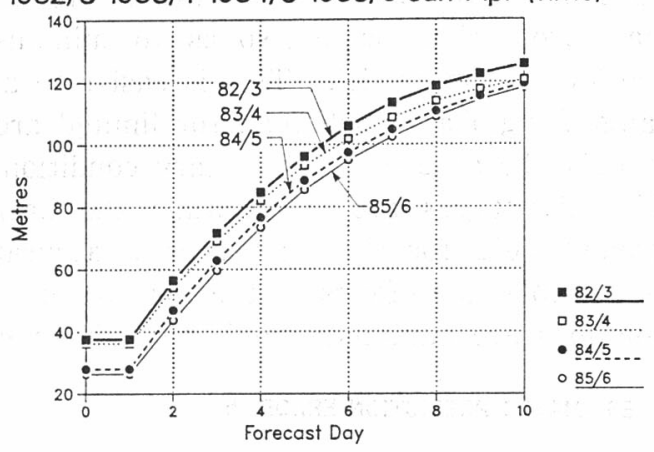

(a)

Northern Hemisphere 500mb RMS Errors 1982/3 1983/4 1984/5 1985/6 Jun-Apr (11mo)

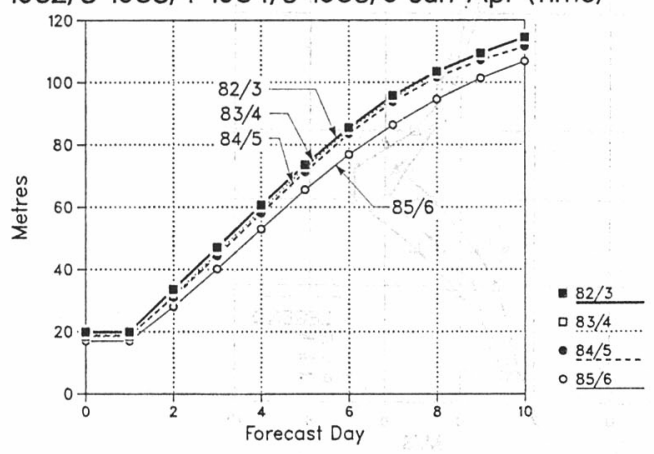

(b)

Fig. 1 RMS $500 \mathrm{mb}$ height forecast errors as a function of forecast range for the Southern hemisphere (a) and the Northern hemisphere (b). The results are shown for the months of June through April in 1982/83, 1983/84, 1984/ $85,1985 / 86$. The one day forecast errors are plotted twice (at day 0 and day 1) to show clearly the effect of successive system changes. Between the first and second periods the model changed from N48 to T63 with envelope orography; between the second and third periods there were substantial changes to the analysis; between the third and fourth periods the model changed to T106 with extensive changes in the physics. See text for further details. (from Shaw et al., 1987).

of the T63 spectral model in 1983 led to a modest gain in forecast skill, as expected (Girard and Jarraud, 1983). The analysis changes in 1984 also gave a modest improvement in medium range forecast skill; we shall see below that the 6 -hour forecasts, and therefore the analyses, were clearly better. The introduction, in 1985, of the T106 model with improved physics led to a marked improvement in Northern Hemisphere forecast skill.

The Northern Hemisphere and Southern Hemisphere results can be interpreted in different ways. Improved analysis methods led to a clear improvement in forecast skill in the data-sparse Southern Hemisphere in 1984/ 85 , before the build up in the number of TOGA drifting buoys from late 1985 onward. The modest impact of the high resolution model on Southern Hemisphere forecast skill may be attributable to the still large analysis uncertainties (Jarraud Simmons and Kanamitsu, 1985). If this is true then analysis error rather than model error is still the dominant contributor to forecast error in the Southern Hemisphere.

In contrast, on the basis of experiments with FGGE data and the 1982 model, Arpe et al. (1985) suggested that model error rather than analysis error was the main source of forecast error in the Northern Hemisphere in the range from two days out to six days and perhaps even further. Model error was defined in this study as the forecast error occurring if the analysis were perfect, and analysis error was defined as the forecast error occurring if the model were perfect. The relative effects of improving the analysis in 1984 and improving the forecast model in 1983 and 1985 suggest that this conclusion was well founded for the 1982 model. Similar experiments with the $1985 / 6$ model would be of interest. This interpretation should be tempered by the fact that the more accurate 1985/6 model probably improved the analyses through the data assimilation cycle (Leith, 1984), as is clearly implied by the marked improvement in the one day forecasts. It would be interesting, but costly, to estimate how much of the improvement in the medium range is due directly to the improved model and how much is due to its indirect effect on analysis quality.

\subsection{The forecast model}

The importance of an accurate forecast model for data assimilation cannot be overemphasised. Accuracy of the forecast model 
is the key to success, as it justifies the simplifying assumptions used in the other three phases: forward interpolation, analysis and initialisation. The smaller the forecast errors, the more linear will be the multivariate relations between the mass field and the wind components, the smaller will be the changes made by the analysis, and the smaller will be the changes made by the initialisation.

From the point of view of Numerical

\section{PERCEIVED ERROR a)}

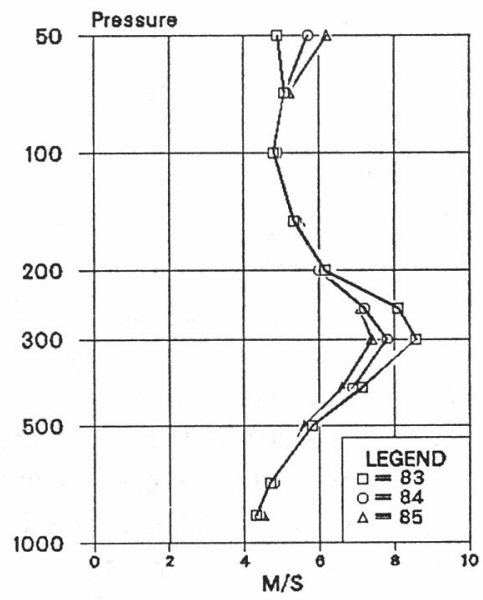

Weather Prediction it is desirable to use the same model for data assimilation and for the main production forecast so as to minimise shocks to the model. This is easier to arrange for global models than for limited area models, because of the boundary conditions. The advantages are substantial: the background field for the analysis is as accurate as possible, the effects of the forward interpolations are minimised, and the adjustment

ESTIMATED PREDICTION ERROR b)

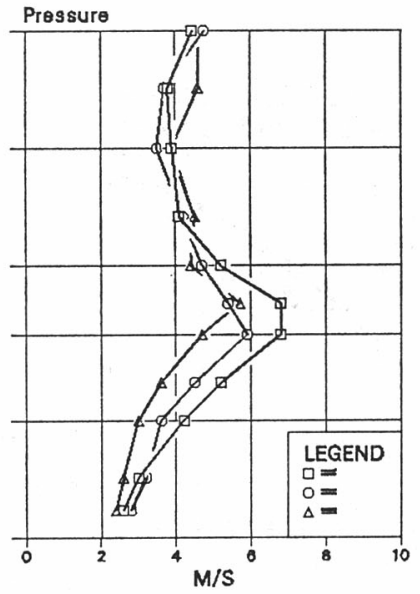

ESTIMATED OBSERVATION ERROR c)

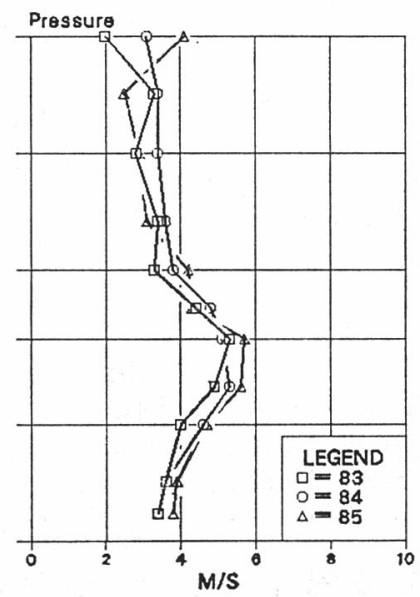

Fig. 2 Perceived wind forecast error (panel a) for 6-hour forecasts verified against 1200 UT North American rawinsondes for Jan-March 1983, JanMarch 1984, and December 1984-February 1985. The perceived forecast error for each year was partitioned into (spatially correlated) prediction error given in panel b, and (spatially uncorrelated) observation error in panel c. Note the stability of the estimates of observation error from year to year, and the steady reduction in the prediction error in the upper troposphere. (from Shaw et al. 1987). 
between physics and dynamics at the start of the forecast, the 'spin-up', is minimised or eliminated entirely.

A most important test of an analysis, for practitioners of Numerical Weather Prediction, is the quality of the ensuing short-range forecast. Apart from operational considerations, this test has the important advantage that the errors of forecast and observations are statistically independent, unless the observations are biased. This test also provides a good evaluation of the internal balance of the analysis because a large gravity wave component in the first guess error is easily detected by the methods discussed in section 6 .

There is an appreciable interaction between the quality of the analysis and the quality of the forecast model. Fig. 2a shows the perceived, or measured, forecast errors in the wind field over North America in the three 3month periods Jan.-Mar. 1983, Jan.-Mar. 1984, and Dec. 84-Feb. 85 as measured by rawinsondes (Shaw et al., 1987). No important changes in radiosonde coverage occurred during the overall period in question. There is a clear reduction in the magnitude of the perceived forecast errors between 400 and $250 \mathrm{mb}$ as we progress from one year to the next. Between the first and second winter there was an important model change. The forecasts for the '83 winter were made with the previously described N48 operational grid-point model. The forecasts for the $83 / 84$ winter were made with the T63 spectral model using the same physical parameterisation package and envelope orography.

Between the second and third winter there was the major change in the analysis, and some changes in the model already described. The forecasts for the $84 / 85$ period were made with the same model as in the $83 / 84$ period except for the introduction of a diurnal cycle, and a modified representation of the radiative transmission functions. It is unlikely that either change had much effect on 6-hour wind forecast errors over North America, and so the gains in forecast skill between the second and third winter were mainly due to the analysis changes.

There is a clear implication from the re- sults in Fig. 2a that both the introduction of the new model in 1983, and the analysis revision in 1984, had important benefits for the accuracy of the short term wind forecasts in the upper troposphere. To document this conclusion more clearly, Fig. 2b shows the estimates of wind prediction error for each of the three periods, calculated as in HL/LH. The improvement in the quality of the shortrange forecasts is quite evident, and demonstrates that both model improvements and analysis improvements can contribute to short range forecast improvements. As a check on the results, Fig. $2 \mathrm{c}$ shows the observation error for wind for each of the three periods, estimated in same way. A certain amount of noise is evident in both Figs. $2 \mathrm{~b}$ and $2 \mathrm{c}$, but the main results are clear-an almost constant level of observation error and a clear improvement in forecast skill in the upper troposphere.

\subsection{The forward calculations}

In the language of geophysical inverse theory (Menke, 1984), the interpolation of the background field to the observation point may be referred to as the 'forward' problem, and the interpolation of the observations back to the model may be referred to as the 'inverse' problem. Historically, most attention in meteorological assimilation has been given to the inverse problem i.e. to the analysis. The perception of the forward problem as a trivial interpolation to the observation position needs to change, because it is critical for a successful analysis, and it is difficult.

For a univariate problem Franke (1985) demonstrates the importance of accuracy in the interpolation of the first-guess to the observation position by showing that the advantage of $\mathrm{O} / \mathrm{I}$ over competing methods can be completely negated if the $\mathrm{O} / \mathrm{I}$ algorithm uses an inferior interpolation scheme for the forward calculation.

Bourke, Seaman and Puri (1985) note that two features of prediction models which influence assimilation systems are the use of terrain-following sigma-coordinates, and the use of temperature as the thermodynamic variacle. They comment that in multivariate 
analysis a sigma coordinate system does not readily allow application of the geostrophic wind law, and temperature is less easily used than is geopotential. Accordingly the analysis component of the assimilation is frequently implemented in pressure coordinates. The model prediction is thus interpolated from model to pressure coordinates, corrected by available data, and the resultant increments or changes interpolated back to the model sigma domain'.

The importance of accuracy in the forward calculation is much greater in such a multivariate problem, since considerations of balance are of central importance in multivariate analysis. Currently operational forms of $\mathrm{O} / \mathrm{I}$ assume that the first-guess is in balance; they then calculate (linearly-) balanced increments from the differences between observations and first-guess. It is quite difficult to make univariate interpolations of the mass and wind components, and still preserve subtle properties such as approximate geostrophy or non-divergence. Wergen and Simmons (1986) have shown that serious consequences can arise when error in the forward calculation generates an unbalanced first guess.

The imbalance in their study arose because mass and wind were interpolated univariately from the model's coordinates to provide a first guess on standard pressure levels. Even though the model was balanced on its hybrid coordinate sigma surfaces (Simmons and Jarraud, 1983), the first-guess on pressure surfaces was seriously unbalanced due to a substantial mis-match between the model's vertical resolution and the set of standard levels on which the first guess was needed for the analysis. In their case the background field was needed at $10,20,30,50,70$ and $100 \mathrm{hPa}$ in the stratosphere, and the original model had the temperature levels at 25, 75 and $130 \mathrm{hPa}$. They compared the first guess generated at $50 \mathrm{hPa}$ from this assimilation, with the first guess from an assimilation where the revised model had levels at approximately $10,30,50,70,100$ and $130 \mathrm{hPa}$. The balance of the first guess was seriously defective in the one case, and quite good in the other.

If the first-guess is unbalanced and the observations are balanced, then the observation minus first-guess deviations will be unbalanced, and will be heavily filtered (section 4). As a result, much useful information will be rejected by the assimilaion because of errors in the forward interpolation. When they ran an extended assimilation with enhanced vertical resolution in the assimilating model, Wergen and Simmons found that there were important improvements in the medium range forecasts as well as in the short range forecasts. They showed that the medium range improvements were largely attributable to the improvement in the initial data.

The work by Wergen and Simmons is the latest of a series of developments at ECMWF aimed at improving the vertical interpolation algorithms. The first implementation of the ECMWF analysis system (Lorenc, 1981), interpolated the first guess from model coordinates to pressure coordinates, evaluated the new analysis fields in pressure coordinates and then interpolated these univariately to model coordinates. A revision to this scheme was introduced by Talagrand in 1980, whereby the analysis increments were interpolated multivariately from the data points to the horizontal projection of the model grid in pressure coordinates. The analysis increments were then interpolated univariately from pressure coordinates to model coordinates in the vertical. This procedure has since been adopted at NMC Washington for their global forecast model (Dey and Morone, 1983) and at NASA's Goddard Laboratory for Atmospheres (Baker et al., 1984) and elsewhere.

Unden (1984) implemented an algorithm where the analysis increments can be interpolated three-dimensionally and multivariately from the observation points to model coordinates, taking account of the usual constraints of near-geostrophy and near non-divergence of the increments. This requires the use of continuous positive-definite representations of the vertical correlation functions, ensuring that all correlation matrices generated with these functions are positive-definite. Implementation of these ideas has improved the multivariate properties of the analysis.

Improvements in the multivariate aspects 
of the forward interpolation can come both from improving the resolution of the prediction model, so that the interpolation to observation position is over a shorter distance, and by improving the order and accuracy of the interpolation. The importance of errors in the forward problem will be highlighted again when we discuss developments in the retrieval of information from the satellite sounders.

\subsection{Convergent evolution in assimilation and initialisation techniques}

It has long been recognised that some concept of balance is essential for meteorological analysis. If the concept is ignored then the introduction of new data may generate noise in the assimilation, resulting in the rejection of very expensive and useful information. The techniques of intermittent assimilation and continuous assimilation both address this problem, but the methodologies of the assimilation methods were initially rather different in several important respects (Bengtsson, 1975).

On the whole, the intermittent methods use more elaborate spatial interpolation methods for the observational data. The methods are often multivariate, they use more observational data in the calculation for a given point, and they use fairly simple concepts of balance, a linear concept in the analysis and a linearisable concept in the initialisation. The continuous insertion methods avoid the explicit imposition of balance concepts, and typically use fewer observational data in the calculation of updates for a given point. The continuous method relies on the model's dynamics and physics to spread out the observational information in such a way as to achieve an accurate and balanced analysis.

As a result of the availability of the FGGE datasets, extensive and detailed comparative studies of the response of analysis systems to particular types of data have been undertaken (Hollingsworth et al., 1985 ; Barwell and Lorenc, 1985; Baede et al., 1985; Bromley et al., 1985; Kashiwagi, 1985 ; Uppala et al., 1985 ; Puri et al., 1985; Atlas et al., 1984). These studies served to underline the relative strengths and weak- nesses of the different methods. As the studies have been extensively reviewed already (ECMWF, 1984; National Research Council 1985), there is no need for great detail here, and a few general comments will suffice.

There is evidence of convergence in the evolution of the assimilation systems (Tribbia, 1982; Williamson and Daley, 1983). The intermittent assimilation systems are trying to incorporate more of the model's physics in the initialisation. Many studies have shown that the adiabatic normal mode initialisation had a serious damping effect on the tropical divergence field. This can be overcome by eliminating some of the damping through selective filters (Puri, 1983; Kitade, 1983), or through the incorporation of the large-scale and slowly varying components of the model's diabatic tedencies in the initialisation technique (Wergen, 1982).

At the same time, it has been found desirable, in some continuous assimilation schemes, to be more explicit in the way in which wind data is spread out in the vertical and balanced with height in the horizontal (Miyakoda et al., 1986; Barwell and Lorenc, 1985). The availability of advanced assimilation systems with different design philosophies has been a valuable stimulus to deeper understanding of the problem as a whole.

The culmination of this trend of convergent evolution will probably be realised through the development of practical fourdimensional variational assimilation methods as proposed by Le Dimet and Talagrand (1986) and Lorenc (1986), and discussed further in Section 3.2.

\section{Theoretical and experimental comparison of analysis methods}

Not only has there been convergence in practical aspects of assimilation, there has also been a growing understanding of the close theoretical relations between many of the methods used for the analysis step of the ssimilation. Theoretical work has shown that analysis methods based on optimality principles are closely related to each other, and a synthesis of the theory of analysis methods is now emerging (para 3.1), The theory of 
$\mathrm{O} / \mathrm{I}$ has an important role in this synthesis.

The adjoint technique discussed in para 3.2 is also based on optimality principles. It offers the prospect of a more effective exploitation of the time history of the atmosphere. The value of a better use of the time history will occur at several points in our later, more practical, discussions. Experimental work confirming that the theoretical advantages of $\mathrm{O} / \mathrm{I}$ can also be realised in practice is discussed in para 3.3

\subsection{The theory of 3-Dimensional multi- variate analysis}

Phillips (1982) argued that a multivariate $\mathrm{O} / \mathrm{I}$ analysis using all the available observational data is 'complete' in the sense that no further information can be extracted from the data through a variational analysis with balance constraints. The underlying continuous multivariate analysis (see section 4) defines the slow mode components (Leith, 1980) of the mass and wind field increments completely, subject to the requirements that 1) the covariances used are appropriate for slow modes, and 2) the forecast is sufficiently accurate that it represents the fast modes to a reasonable degree. Phillips (1982) argues that one should use as much data as possible in a single calculation, and that one should analyse as large an area as possible with that data, ideally the whole globe. The box technique developed by Lorenc (1981) therefore finds solid support from theoretical arguments. Lorenc (1986) comments that a limited or restricted data selection is appropriate when the background field has little useful prior information. When the background field is accurate, then there is much to be gained from an extensive data selection, provided the statistics of forecast error are reasonably well known.

Ikawa (1984a, b, c) has made a detailed investigation of the relationship between variational analysis as proposed by Sasaki (1959, 1970 ) and the multivariate O/I algoritm. He confirms Phillips' result on the essential equivalence of the slow-mode $\mathrm{O} / \mathrm{I}$ analysis and the variational analysis. Ikawa first shows how weak and strong constraints can be formulated in essentially similar ways (Ikawa, 1984a). He then shows that the slow-mode covariance matrix as used in $\mathrm{O} / \mathrm{I}$ has the same sort of filtering properties as the variational analysis (Ikawa, 1984b, c).

Lorenc (1986) has taken the synandsis of analysis methods a stage further by demonstrating that $\mathrm{O} / \mathrm{I}$ can be derived by variational arguments, as also shown by Ikawa, and that $\mathrm{O} / \mathrm{I}$ can be derived by Bayesian methods, provided the statistics are Gaussian. We therefore have three independent demonstrations of the equivalence of $\mathrm{O} / \mathrm{I}$ and constrained variational analysis. These results are extensions of earlier work by Kimeldorf and Wahba (1970).

Lorenc (1986) formulates the analysis problem in a very general way. The observations, of themselves, are incapable of defining the state of the atmosphere. Prior information in the form of model equations, or a model forecast, or climatology, or requirements of smoothness, or some combination of these, is needed to resolve the under-determinacy. The analysis problem is posed as the minimisation of a penalty function consisting of terms depending on the distance of the analysis from the data, and on the distance of the analysis from the prior information, each distance being weighted by the accuracy of the corresponding information source. A particular feature of Lorenc's derivation is the way in which any number of weak constraints can be incorporated into a single penalty function.

Lorenc goes on to demonstrate the relationship between $\mathrm{O} / \mathrm{I}$ and the smoothing spline, or thin-plate spline, approach of Wahba and Wendelberger (1980). In the latter approach no information on the mean field (or its first few derivatives) is taken from the first guess. Both the curvature of the interpolating function and the noise level of the observations are adjusted by Bayesian calculations which maximise the likelihood of the final solution. This method is most applicable when no first guess is available, or when the first-guess has little useful information on the larger scales. As shown by Hollingsworth et al. (1986) the first guess produced by a good forecast model 
has much valuable information.

The main difference between the results using continuous correlation functions on the one hand, Phillips (1982) and section 4 below, and those presented by Ikawa (1984) and Lorenc (1986) on the other, is that the latter authors have used a discrete formulation for the spatial dependence of the correlation functions. The use of a discrete formulation has theoretical and practical advantages, since finally the continuous representations used here and in Phillips' discussion must be discretised for the forecast model. As noted by Hollingsworth (1984) the difference between the differential gradient and the finite-difference gradient can be large. However it is not clear from the work of either Ikawa or Lorenc how to determine the discrete correlation representation from observational data. This is likely to be a tricky problem in the multivariate case.

\subsection{More effective incorporation of the time dimension}

There are a number of outstanding problems in analysis which will probably yield only to methods which make more effective use of the time history of the atmosphere. Examples include situations where the state of atmospheric balance is strongly non-linear, as it is near sharp fronts, and the determination of the planetary scales in the Tropics from scattered sparse observations. In these cases the observations available at a single synoptic time are quite inadequate to define the essential structure.

The general analysis framework proposed by Lorenc (1986) can accommodate 4-dimensional as well as three dimensional analysis. When the forward problem is linear, Lorenc shows that the optimal 4-dimensional analysis reduces to a sequence of 3-dimensional analyses, using the forecast from each to provide the background for the next analysis. This is the Kalman-Bucy filter (Ghil et al., 1981). There is a close analogy between approximations to this process and the implementation of many operational intermittent-insertion assimilation systems. The process of initialisation can be incorporated explicitly in the
Kalman-Bucy formalism (Ghil et al., 1981). Alternatively, it can be imposed implicitly through a linear weak constraint that the analysis should mainly consist of slow modes. A major advantage of the Kalman-Bucy approach is that it predicts the error covariances of the background field. This is likely to be very important in regions of sporadic data coverage. Implementation of the full KalmanBucy formalism is expensive because one needs to deal with matrices whose order is given by the model's degrees of freedom.

Le Dimet and Talagrand (1986), exploiting ideas of Marchuk (1975), formulate the fourdimensional assimilation problem in a variational form. Their approach requires the minimisation of a four-dimensional penalty function which includes the model equations as a constraint, a problem which poses formidable difficulties. Le Dimet and Talagrand (1986) propose calculating the gradient of the penalty function with respect to the control variables by integrating the model forward in time for $\mathrm{N}$ time-steps, remembering the model's trajectory at each time level, and then integrating the adjoint model backward in time, using the tanget plane trajectory in the adjoint calculation. One can then determine the direction of descent for minimisation of the penalty function. Iteration of this procedure gives an optimal trajectory over the period of assimilation. If one wishes to start a forecast at day 0 , with an assimilation period of 1 day beforehand, then the starting analysis at day -1 is repeatedly adjusted so as to bring the model evolution as close as possible to the observed evolution during the assimilation period. In this way not only will the atmospheric state at day 0 be well analysed; its tendency, or time derivative, will also be well represented. In a sense the method is a rigorous way to achieve the same goal as the simpler but unsuccessful schemes of the late 1960s such as the forwardbackward scheme (Miyakoda and Mayer, 1968) and the Tadjbakhsh (1969) scheme discussed by Phillips (1971).

Talagrand (1985) and Courtier (1985) have applied the method to a one-level primitive equation model with encouraging results; 
Lewis and Derber (1985) have made similar calculations with filtered models. Extension of the work to multi-level models will undoubtedly follow. A major difficulty will be the treatment of non-differentiable processes, such as convection, for which a suitable adjoint cannot be defined. Nevertheless, the preliminary results are so encouraging, and so many interesting possibilities exist for approximate treatment, that this will be an area of much interest and activity.

On a much simpler level, synopticians make considerable use of the surface pressure tendency field for analysis and forecasting. Extension of the $\mathrm{O} / \mathrm{I}$ formalism to include tilted structure functions for mass and streamfunction, coupled with the divergent wind field, may permit an effective use of pressure tendency data within the O/I formalism. The potential value of such data has been discussed by Bengtsson (1978) in the context of analysing developing systems.

\subsection{Experimental comparison of methods for univariate analysis}

Seaman (1983) and Seaman and Hutchinson (1985) have compared the accuracy of different univariate analysis algorithms using a 20 -year record of surface pressure reports in S.E Australia, with climatology as a background field. They investigated Statistical Interpolation, The Successive Correction Methods of Cressman (1959), and Barnes (1964), Laplacian Spline Fitting with Generalised Cross-validation (Wahba and Wendelberger, 1980), and Statistical Interpolation with Generalised Cross Validation to determine the noise to signal ratio of the observations being analysed. They found that the best results were obtained by statistical interpolation using appropriate forms of correlation function. However the Cressman successive correction method was only slightly less accurate than statistical interpolation with the best functions, and was better than statistical interpolation with a Gaussian correlation function. They also found some suggestion that when the covariance structure is not well known, a modest improvement in statistical interpolation accuracy may be achievable by changing the 'noise to signal' parameter from case to case, using Laplacian spline fitting with generalised cross validation.

Franke (1985) has examined the errors in objective analysis arising from two sources, the interpolation of the gridded first guess to the observation position, and the interpolation of the observation minus first guess information from the observation points back to the grid. His results on the second question, the observation-point to grid interpolation, are in general agreement with those of Seaman (1983) and Seaman and Hutchinson (1985) in showing that the $\mathrm{O} / \mathrm{I}$ approach has an edge over most other methods in univariate problems with uniform observation errors. Franke's results on the importance of the forward calculation have been noted already. The final point made by Franke is that $\mathrm{O} / \mathrm{I}$ is more successful at making an analysis than at estimating the analysis accuracy. The reason is that the calculation of the second moment of the analysis error makes no allowance for error in the assumed covariances of forecast error.

These extensive tests show that $\mathrm{O} / \mathrm{I}$ is better than competing methods on univariate problems, with observations of known uniform quality. In operational analysis one deals with more complex problems of multivariate analysis with data of variable quality. The theory of statistical interpolation suggests that a properly tuned $\mathrm{O} / \mathrm{I}$ analysis would do even better than the competing methods in such circumstances (Seaman, 1977). The methods for analysis verification discussed in section 7 provide a framework in which to compare the performance of different analysis methods in a multivariate context; comparative studies of this kind have yet to be made.

\section{4. $0 / \mathrm{I}$ as a filter and an interpolator}

The basic task of any analysis method is to filter as much noise as possible from the observations, and to interpolate the information to a model grid as accurately as possible. Since the $\mathrm{O} / \mathrm{I}$ algorithm plays a central role in theoretical discussions of analysis method, it is worthwhile to present a simple interpretation of the filtering and interpolation 
properties of the algorithm. It is always helpful in scientific problems to have an intuition about how the solution will look, and it is not easy to develop intuition about the inverses of large matrices; the results presented below may help.

The results of para 4.1 provide a complete description of the response characteristics of the $\mathrm{O} / \mathrm{I}$ system when all available data has been used by the algorithm. This is normally impractical. An approximation to the ideal data selection was introduced by Lorenc (1981), based on a box method. The response characteristics of this algorithm are discussed in para 4.2 following the work of Daley (1985). The effect of correlated observational error on the $\mathrm{O} / \mathrm{I}$ filter is discussed in para 4.3 .

\subsection{The connection between filtering and interpolation in $\mathrm{O} / \mathrm{I}$}

As demonstrated in many papers (Gandin, 1963; Rutherford, 1972 ; Schlatter, 1975, Bergman, 1979; Lorenc, 1981) the O/I analysis equation may be written in the form

$$
a\left(r_{k}\right)=\left[p\left(r_{k}, r_{i}\right)\right]^{*}(\boldsymbol{P}+\boldsymbol{D})^{-1} \cdot\left[d\left(r_{i}\right)\right]
$$

where $r_{i}$ indexes the position and the observed variable at the observation points;

$r_{k}$ indicates the position and variable at the analysis point (it is usually convenient to treat the spatial dependence as continuous);

$\left[d\left(r_{i}\right)\right]$ is the vector of differences between the observed and background values normalised by the standard deviation of the background error; we shall speak of these quantities as the observation minus first-guess (Ob-Fg) differences; the notation [] , or a single underline, will be used for a vector;

$a\left(r_{i}\right)$ is the difference between the analysed and background values normalised by the standard deviation of the background error ;

$\left[p\left(r_{k}, r_{i}\right)\right]$ is the vector of background error correlations between the observed variables at points $r_{i}$ and the analysed variable at point $r_{k}$, and the asterisk indicates transpose ;

$P$ is the correlation matrix of the back- ground field error for the observed variables at points $r_{i}$.

$\boldsymbol{D}$ is the corresponding observational error covariance matrix, normalised by the variance of the background field error; and all variables are assumed to be unbiased.

We may write the vector of analysed values at the observation points as

$$
\left[a\left(r_{i}\right)\right]=\boldsymbol{P} \cdot(\boldsymbol{P}+\boldsymbol{D})^{-1} \cdot\left[d\left(r_{i}\right)\right]
$$

so that Eq. 4.1 may be rewritten as

$$
a\left(r_{k}\right)=\left[p\left(r_{k}, r_{i}\right)\right]^{*}(\boldsymbol{P})^{-1} \cdot\left[a\left(r_{i}\right)\right]
$$

Thus the analysis calculation may be thought of as a linear filter (Eq. 4.2) of the observed values to produce the analysed values at the observation points, followed by an interpolation (Eq. 4.3) of the filtered observations to the analysis points.

The nature of the filtering and interpolation are discussed in the appendix. Here we discuss the special case where $\boldsymbol{P}$ and $\boldsymbol{D}$ commute. This is a good approximation to many cases of practical importance, such as $\boldsymbol{D}=\boldsymbol{\sigma} \boldsymbol{I}$, when the observation errors are random, uniform, and independent.

When the matrices $\boldsymbol{P}$ and $\boldsymbol{D}$ commute, the relation between the filtering and the interpolation is very simple. As shown in the appendix, if the original vector $\left[d\left(r_{i}\right)\right]$ is expanded in eigenvectors $\boldsymbol{e}_{j}$ of $P$ as

$$
\left[d\left(r_{i}\right)\right]=\sum_{1}^{N} c_{j} e_{j}
$$

then the analysed values at the observation points are given by

$$
\left[a\left(r_{i}\right)\right]=\sum_{1}^{N} c_{j} \frac{1}{1+\nu_{j}} \boldsymbol{e}_{j}
$$

where $\nu_{j}$ is the ratio of observational error to prediction error in the $j$ th component. The analysed value at the point $r_{k}$ is given by

$$
a\left(r_{k}\right)=\sum_{1}^{N} c_{j} \frac{1}{1+\nu_{j}} \mathcal{E}_{j}\left(r_{k}\right)
$$

where the function $\mathcal{E}_{j}$ interpolates the vector $e_{j}$ according to Eq. 4.3. The simple relation between the data representation (Eq. A.3), the representation of the filtered data (Eq. 
A.4) and the representation of the continuous multivariate analysis (Eq. A.5) is aesthetically pleasing, and provides a simple insight into the way the $\mathrm{O} / \mathrm{I}$ analysis algorithm works.

The $\mathrm{O} / \mathrm{I}$ algorithm is a filter of the observations, relative to the eigenvectors of the weight matrix $\boldsymbol{P}(\boldsymbol{P}+\boldsymbol{D})^{-1}$, followed by an interpolation with a set of continuous functions which are interpolators of the eigenvectors of the prediction error correlation matrix $\boldsymbol{P}$. In many cases of practical importance the eigenvectors are identical, and the algorithm then has a particularly simple representation (Eqs. A.3-A.5).

For a scalar field with a gaussian correlation, the filter has simple scale dependent properties (Daley, 1985). If one introduces constraints of non-divergence or geostrophy, the multivariate filter will selectively damp divergent or antigeostrophic components in the data, as well as retaining its general scale-dependent character (Daley, 1985). The existence of observational error correlations can substantially modify the scale selectivity properties. These topics will be discussed presently. The continuous multivariate fields of analysis incements produced by the analysis A. 5 have all the properties of differentiability, scale dependent geostrophy and scale dependent non-divergence that one wishes to impose. When the analysis increment is evaluated on a finite difference mesh, or in a truncated spectral representation, these desirable properties may be retained only in an approximate fashion.

\subsection{Scale dependent response of the $O / I$ algorithm}

An implementation of the $\mathrm{O} / \mathrm{I}$ system using all the data available at a given time would provide a multivariate and three-dimensiona] interpolation of the observational data satisfying all the requirements of differentiability, linear balance and near non-divergence one wishes to impose. This ideal will remain a dream for some years, and it is necessary to make intelligent approximations to the ideal. One such approximation was devised by Lorenc (1981), using a box method for the analysis.
The results of Phillips (1982) and Lorenc (1986) provide theoretical support for the box approach to $\mathrm{O} / \mathrm{I}$ analysis introduced by Lorenc (1981). Seaman (1977), Lönnberg (1982), Andersen (1982) and Daley (1983, 1985) addressed theoretical and practical questions concerning the response of the box-based analysis system to data for different variables, and to data on different scales. Early experience with the ECMWF system indicated that the system had difficulty in analysing small scale rapidly developing disturbances. It was not clear which features of the system were responsible for these defects. It has since emerged that several aspects of the system needed refinement, including both the horizontal and vertical resolution. A good theoretical basis and a statistical database were needed to address these questions.

As formulated by Lorenc (1981) the analysis algorithm selects data on mass and wind for up to 9 levels within and around each analysis box of about $660 \mathrm{~km}$ on a side. A multivariate analysis is then performed for mass and wind at all the grid-points and levels within the box. A refinement to the algorithm known as the 'box overlap' was introduced by Andersen in 1981. In this refinement the analysis for each box is evaluated for a region extending outside the box to the centre of the neighbouring boxes. This provides several additional estimates of the analysis for each box. These are combined in a simple weighted mean. It was this version of the analysis that was examined by Daley (1983, 1985).

To study the scale dependence of the response, Daley sampled simple sinusoidal functions with a regular array of observing points, and examined the magnitude of the response as a function of the observation spacing and the scale of the disturbance. For these experiments the geopotential auto-correlation was an idealised Gaussian with a length scale of $600 \mathrm{~km}$, the geostrophic coupling coefficient was 0.95 , and the wind structure function was strictly non-divergent.

Fig. 3 summarises his results. Fig. 3a shows the response (ratio of output amplitude to input amplitude) in the geopotential field 


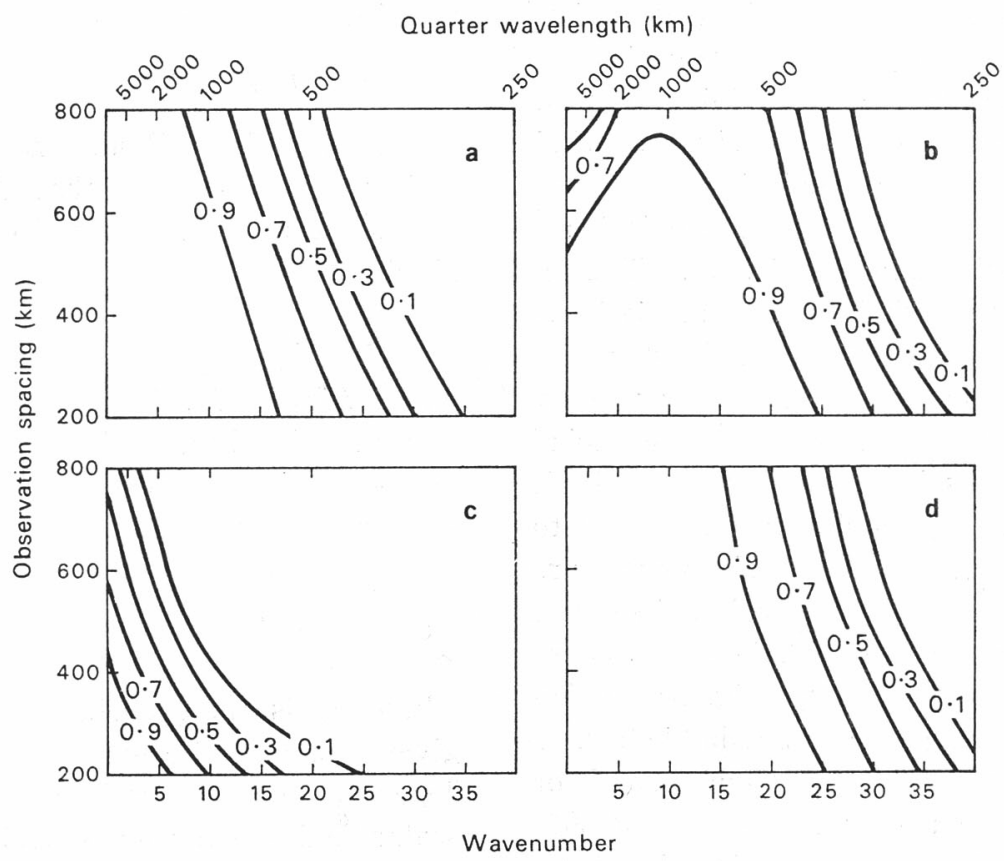

Fig. 3 Response diagrams illustrating response of a box-type analysis to regularly spaced observational data, as a function of input wavenumber (abscissa) and observation spacing (ordinate). On the top of each diagram is also shown the quarter wavelength $(\mathrm{km})$. a) geopotential response to geopotential observations. b) wind response to non-divergent wind observations. c) wind response to irrotational wind observations. d) mass, or wind, response to mass and non-divergent wind observations. The auto-correlation functions are modelled by a gaussian with a $600 \mathrm{~km}$ length scale, and the non-dimensional observation error squared is 0.25 in all cases (from Daley, 1985).

to geopotential observations. There is a marked sensitivity of response to both the scale of the input and the observation spacing. For observation spacings larger than $200 \mathrm{~km}$ the response is less than 10\% for planetary wave-numbers higher than 35 (quarter wave-length $=285 \mathrm{~km}$ ); for observation spacings larger than $300 \mathrm{~km}$ the response is less than $30 \%$ for planetary wave-numbers higher than 25 (quarter wave-length $=400$ $\mathrm{km})$.

The two-dimensional non-divergent wind response to non-divergent wind observations in Fig. $3 \mathrm{~b}$ is rather better than the height response, with improved response at all wavenumbers between 10 (quarter wave-length $=1000 \mathrm{~km}$ ) and 35 . However there are clear indications of problems in the analysis of the very largest scales when the observation spacing is equal to or larger than the length scale of the Gaussian (see upper left corner of Fig. 3b). This has been remedied by recognising that the correlation function must include a representation of forecast errors which are of such large scale that they are essentially constant over the region for which analysis calculations are made (HL/LH). The square of the normalised observation error was 0.25 in both Fig. $3 a$ and Fig. $3 b$ so the extra response on synoptic scales in the wind field must come from the non-divergence constraint and the fact that two wind-components are analysed simultaneously.

The effect of analysing height or wind using both height and wind observations is shown in Fig. 3d. Comparing Fig. 3d with $3 a$ shows that the presence of the wind data improves the height analysis on short scales, 
while the presence of the height data improves the wind analysis on large scales.

In general terms one may think of Fig. 3d as the mid-latitude response to height or wind data, Fig. 3a as the tropical response to height data, and Fig. 3b as the tropical response to non-divergent wind data. The reponse to divergent wind data at any latitude is shown in Fig. 3c. Although the structure functions are non-divergent, they are only applied in local calculations with non-global data selection. As discussed by Lorenc (1981, 1986), it is then possible for the system to respond to divergent wind fields which are representable on a 6-degree grid, and so have a minimum wave-length of 12 degrees. These arguments are borne out by Fig. 3c, which shows that the response is negligible beyond wavenumber 25. For wave-number 5 or lower there is a response in excess of $30 \%$ provided the observation spacing is closer than $600 \mathrm{~km}$.

These results are dependent on the width of the Gaussian correlation function used for the calculations. The spectral representation of forecast error discussed in section 6 offers a general approach to the problem of improving resolution at small scales while maintaining good response characteristics at large scales. Simple calculations provide a valuable check on the properties of any proposed revisions of the correlation function.

\subsection{The effect of correlated observation error}

In many applications the observations have correlated errors (Schlatter, 1981). A correct treatment of these errors can change the filtering effect of the $\mathrm{O} / \mathrm{I}$ algorithm. Once the filtering step is complete, the observation error correlations have no further effect on the interpolation step. The role of correlated observational error has been discussed by Bergman and Bonner (1976) and by Seaman (1977) who argue that the presence of correlated error in mass-field observations can effectively improve the resolution of the analysis on small scales. Hollingsworth, Lönnberg and Andersen, (pers comm. 1987), show that one may gain useful insight on the problem by considering the filtering step of the $\mathrm{O} / \mathrm{I}$ algorithm. As noted previously

$$
[a]=\boldsymbol{P}(\boldsymbol{P}+\boldsymbol{D})^{-1} \cdot[d]
$$

where $[a],[d], \boldsymbol{P}$ and $\boldsymbol{D}$ are as defined earlier. Suppose first that the observation errors are uncorrelated and of uniform magnitude

$$
D=\sigma I
$$

where $\boldsymbol{I}$ is the identity matrix. If the original data and the filtered data are expanded in terms of the eigenvalues of $P$, then the response $R_{i}$ to the $i$ th component is

$$
R_{i}=\frac{\lambda_{i}}{\lambda_{i}+\sigma}=\frac{1}{1+\frac{\sigma}{\lambda_{i}}}
$$

where $\lambda_{i}$ is the $i$ th eigenvalue of $\boldsymbol{P}$. This gives a simple filtering of the observations depending on the ratio of $\sigma$ to $\lambda_{i}$. For a scalar field like the mass field this translates readily into a scale-dependent filter.

Suppose now that the observations have a correlated error, and that the error can be represented as the first two terms in a power series expansion in $\boldsymbol{P}$ :

$$
D=\sigma I+\varepsilon P
$$

where we shall speak of the terms on the right as the un-correlated and correlated parts of the observation error respectively. Empirical support for such a representation of the vertical error correlation of TOVS data can be had by comparing the results of Schlatter (1981) with those of LH for thickness forecast errors. It follows that the response in the $i$ th component in this case becomes

$$
\begin{aligned}
R_{i} & =\frac{\lambda_{i}}{(1+\varepsilon) \lambda_{i}+\sigma} \\
& =\frac{1}{1+\varepsilon} \cdot \frac{1}{1+\frac{\sigma}{(1+\varepsilon) \lambda_{i}}}
\end{aligned}
$$

The presence of correlated observational error has two effects. Firstly it damps all components uniformly by an amount $1 / 1+\varepsilon$. Secondly it reduces the scale-selective damping of the algorithm by reducing the term $\sigma / \lambda_{i}$, which controls the scale selectivity in Eq. 4.4, to $\sigma /(1+\varepsilon) \lambda_{i}$. If there is no uncorrelated error as defined above, then the re- 
sponse is simply $1 / 1+\varepsilon$. The filter is no longer scale- selective, and all components of the data are treated equally.

In this limiting case the data is treated as if it were exact, except for the uniform factor $1 / 1+\varepsilon$; this occurs because of the relative magnitudes of first-guess error (normalised to 1) and observation error, given that both have the same spatial correlation. This result indicates that care is needed in the specification of the observation errors. Polar orbiting satellite temperature retrievals are well known to have both vertically and horizontally correlated observation errors, arising mainly from the retrieval techniques rather than from the original radiance measurements. Current estimates of forecast error structure (HL/LH) and observation error structure for satellite retrievals (Kelly, 1985) indicate that the associated length scales are not too dissimilar, so the above simple example is relevant for practical work.

\section{A framework for verification of observations, forecasts and analyses}

The particular strengths of the O/I algorithm are the ease with which it can handle heterogeneous observations, and the simplicity of the multivariate formulation. If constraints such as near-geostrophy or non-divergence are imposed on the covariance functions, they will be satisfied by the continuous analysis. The ability of the O/I system to cope with correlated observational error is important in practice. The central role played by the error characteristics of the observations and of the first-guess in the $\mathrm{O} / \mathrm{I}$ filtering and interpolation indicates that one must determine these statistics as accurately as possible.

In the next few sections we discuss a general approach to the determination of the statistical structure of atmospheric fluctuations, of forecast errors and of analysis errors. The kinematics of two-dimensional turbulence provide the connecting link between all three discussions. Having outlined the basis of the techniques we discuss

i) the statistical properties of atmospheric fluctuations as seen by different observing systems (section 5.2) ii ) the statistical structure of short-range forecast errors (section 6), and

iii) the verification of analyses (section 7).

Most of the results are concerned with the isotropic component of the statistics; the anisotropic components of the statistics are briefly discussed in section 6.3 .

\subsection{Two-point correlations and the kinematics of two-dimensional turbulence}

The kinematic theory of 2-point velocity correlations in homogeneous conditions was worked out in the 1930s and 1940s by workers on turbulence theory. The results are summarised in standard texts (Batchelor, 1953; Panchev, 1972). These results were used by Hutchings (1955), Buell, (1972), Brown and Robinson (1979), in discussions of the statistical structure of fluctuations about the climatological norm.

Daley (1985) used the kinematic equations of 2-dimensional turbulence in a Cartesian framework to formulate the $\mathrm{O} / \mathrm{I}$ analysis problem for mass and wind in a plane, with no constraint of non-divergence. HL/LH (1986), used the kinematic equations in the coordinate system commonly used in turbulence theory to estimate the three auto-correlations $\langle\phi, \phi\rangle$, $\langle\psi, \phi\rangle,\langle\chi, \chi\rangle$ and the three cross-correlations $\langle\phi, \phi\rangle,\langle\phi, \chi\rangle,\langle\phi, \chi\rangle$, where $\phi, \phi, \chi$ represent the fluctuations of geopotential, streamfunction and velocity potential.

Assuming homogeneous conditions, and defining

$$
\begin{aligned}
& F(r, \theta)=\gamma^{2}\langle\psi, \phi\rangle \\
& G(r, \theta)=\delta^{2}\langle\chi, \chi\rangle \\
& H(r, \theta)=\gamma \delta\langle\psi, \chi\rangle
\end{aligned}
$$

where

$$
\begin{aligned}
& \gamma=E_{\psi} / E_{l} ; \\
& \delta=E_{\chi} / E_{l} ;
\end{aligned}
$$

and $E_{\psi}, E_{\chi}$ and $E_{l}$ are the standard deviations of the $\psi, \chi$ and velocity component fluctuations, the kinematic equations for the winds may be written 


$$
\begin{aligned}
& -\nabla^{2}(F+G)=\langle l, l\rangle+\langle t, t\rangle \\
& L_{1}(F-G)-4 L_{2}(H)=\langle l, l\rangle-\langle t, t\rangle \\
& L_{1}(H)+L_{2}(F-G)=-\langle l, t\rangle
\end{aligned}
$$

where $l, t$ denote the velocity components along (longitudinal) and perpindicular to (transverse component) the line joining two observation points. Using polar coordinates $(r, \theta)$ for the displacement between two stations, the operators in Eq. 5.1 have the form

$$
\begin{aligned}
& \nabla^{2}=r^{2} R^{2}+2 R+T^{2}, \quad R=\frac{1}{r} \frac{d}{d r}, \\
& T=\frac{1}{r} \frac{d}{d \theta}, \quad L_{1}=r^{2} R^{2}-T^{2}, \\
& L_{2}=T\left(\frac{1}{r}-r R\right) .
\end{aligned}
$$

Solutions of these equations may be found from empirical data on the velocity correlations by least squares fitting procedures using expansions in Fourier-Bessel series.

Only the isotropic terms have been studied by $\mathrm{HL} / \mathrm{LH}$, and by most earlier investigators. In isotropic conditions the equations $5.1 \mathrm{sim}$ plify to

$$
\begin{aligned}
& -\left(r^{2} R^{2}+2 R\right)(F+G)=\langle l, l\rangle+\langle t, t\rangle \\
& r^{2} R^{2}(F-G)=\langle l, l\rangle-\langle t, t\rangle \\
& -r^{2} R^{2} H=\langle l, t\rangle
\end{aligned}
$$

If the turbulence is non-divergent then $H=G$ $=0$, and the equations simplify to

$$
\begin{aligned}
& -R F=\langle l, l\rangle \\
& -\left(r^{2} R^{2}+R\right) F=\langle t, t\rangle
\end{aligned}
$$

which implies a well known relation between the longitudinal and transverse velocity correlations in homogeneous isotropic incompressible flow (von Karman and Howarth, 1938). The equivalent relation between the longitudinal and transverse velocity correlations in purely divergent flow were derived by Obukhov (1954), in his thesis. Obukhov commented that he could not imagine an application which would need to consider flows which were partly divergent!

HL/LH found empirical expressions for $\langle\psi, \phi\rangle,\langle\chi, \chi\rangle$ and $\langle\psi, \chi\rangle$ by substituting spectral expansions for these quantities in the left hand sides of Eq. 5.2, and making a least squares fit of the resulting expressions to the empirical data on the right.

The determination of the cross-correlations of mass and wind is rather simpler than the wind-wind correlations, as it involves the solution of a pair of coupled first-order linear partial differential equations rather than a set of three coupled second-order PDEs. If $I(r, \theta)$, and $J(r, \theta)$ are defined by

$$
\begin{aligned}
& I(r, \theta)=\gamma\langle\phi, \phi\rangle \\
& J(r, \theta)=\delta\langle\phi, \chi\rangle
\end{aligned}
$$

then for homogeneous conditions

$$
\begin{aligned}
& I_{r}+\frac{1}{r} J_{\theta}=-\langle\psi, t\rangle \\
& J_{r}-\frac{1}{r} I_{\theta}=-\langle\phi, l\rangle
\end{aligned}
$$

If conditions are isotropic then the equations are especially simple:

$$
\begin{gathered}
I_{r}=-\langle\phi, t\rangle \\
J_{r}=-\langle\phi, l\rangle
\end{gathered}
$$

These last equations have a simple physical interpretation if we consider the correlation of the geopotential at one radiosonde location with the radial and transverse wind components at surrounding stations. The height/ streamfunction correlation is defined by the correlation between the geopotential and the swirl velocity component about the geopotential observing point, while the height/ velocity-potential correlation is defined by the correlation between the geopotential and the radial velocity component. The $\langle\phi, \phi\rangle$ correlation is determined directly from observations using the methods of Rutherford (1972).

\subsection{Intercomparison of observing systems}

There have been extensive studies of the analysis of atmospheric fluctuations, using climatology as a background (Gandin, 1963; Rutherford, 1972 ; Buell, 1971 ; Buell, 1972a, b ; Panchev, 1971; Julian and Thiebaux, 1975; Brown and Robinson, 1979 ; Buell and Seaman, 1984 ; Rinne and Jarvenoja, 1985). Several of these studies have used the simplified non- 
divergent form of the kinematic equations to investigate atmospheric fluctuations.

There is however a further application of the methods which is suggested by Wylie et al. (1985), who raise the important question: Do different observing systems see the same atmosphere? They studied the spatial correlation structure of the fluctuations of the tropical wind field about the long term mean, using the vector wind correlation $\langle l, l\rangle+$ $\langle t, t\rangle$, the spatial correlation for wind speed, and Gandin structure functions for wind speed. They compared the statistics derived from rawinsondes, aircraft, and cloud track winds. Fig. 4 shows an example of their results for the vector wind in the tropical upper troposphere. It shows that there are some striking differences between the statistics of atmospheric fluctuations as derived from different observing systems, indicating that the observing systems sample quite different scales of motion (cf. the correlations for automatic aireps and radiosondes). The very broad correlations for the cloud track wind data suggest that the data sample only large scales. Relative to radiosonde winds, the cloud track wind data therefore have correlated sampling errors.

Further work along these lines would be valuable. Comparison of spectra from observation systems with uncorrelated errors

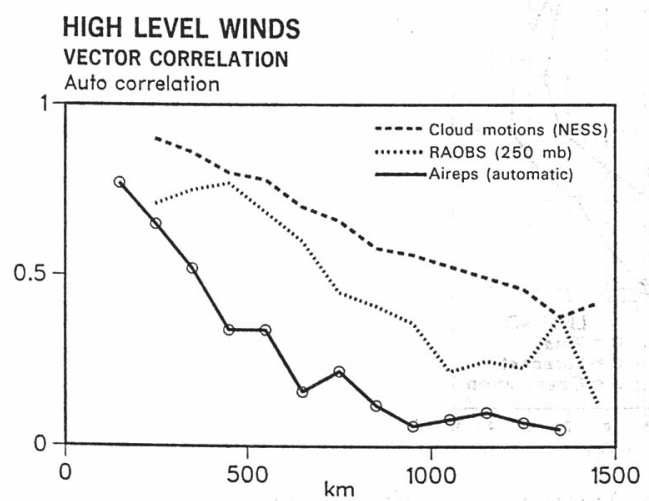

Fig. 4 Tropical $250 \mathrm{mb}$ vector wind correlation of fluctuations about the climate mean, as observed during FGGE by three observing systems The aircraft sample much shorter scales than the radiosondes, and the cloud track winds sample much larger scales. (from Wylie, Hinton Howland and Lord, 1985). (e.g. rawinsondes and aircraft) would determine a spectral band where they both have equivalent response. It should then be possible by cross comparison with satellite data to quantify the structure of spatially correlated observation errors, giving a different approach from Schlatter (1981). One could, for example, also address the question of 'Do different wind observing systems have the same response to divergent flow?' by examination of the statistics on divergent flow resulting from solutions of Eqs. 5.1 or Eqs. 5.2 , where the empirical data is derived from different observing systems. Such information would be quite important for tropical analysis.

\subsection{Data monitoring}

Besides the elaborate methods just discussed for evaluation of observing systems, there are much simpler techniques which are also valuable. As discussed in section 8, every operational system needs quality control algorithms to reject data which may be corrupt for one reason or another. Studies of the interaction of data with the assimilation system can be valuable in identifying systematic defects in data.

\section{a) Real time data Monitoring}

An operational data assimilation system needs defences against data which have been corrupted for one reason or another. These defences serve a useful purpose by indicating the data which have problems, and need examination. Several tools besides rejection lists are available to identify data which need examination; maps of analysis increments and regional plots of the rms fit of the analysis and first guess to the data are both heavily used (Delsol, 1985).

In case of real time problems there is a typical sequence of investigations. The first concern is to make sure that the data have been correctly treated in the pre-analysis phase (correct decording, proper climatological tests, etc.) Next there are checks that the differences between the data and the analysed fields are reasonable from the synoptic point of view. An analysis scheme designed for 
models with mesh sizes of $150-200 \mathrm{~km}$ cannot resolve the core of a hurricane; data indicating the central pressure of an intense hurricane will then be rejected on the reasonable scientific grounds that they represent something the analysis and the forecast model cannot resolve. Similarly, data may be rejected by the analysis if they are contradicted by adjacent data of good quality.

In many cases it can take just a short time

a) WIND / NORTH AMERICA

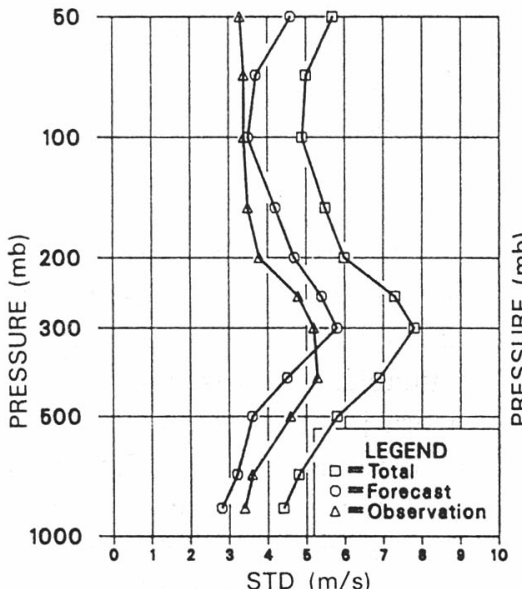

to establish that either the assimilation system or the data have real problems. In other cases it can take several days to establish whether or not the treatment of a particular piece of data or type of data was correct. Depending on the outcome of the investigation, action will need to be taken, either by liaison with the builders of the assimilation system, or by liaison with the data producers in case of a new bug in an observational

\section{b) WIND / EUROPE}

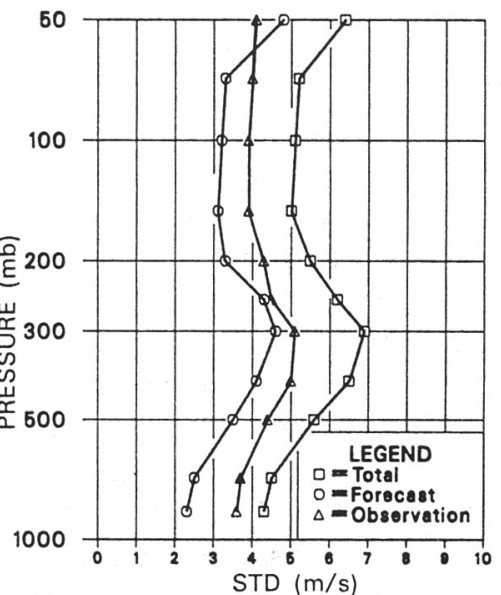

c) WIND / TROPICS

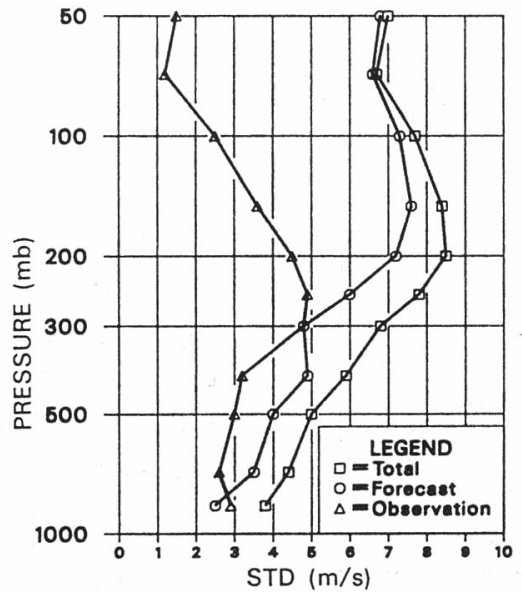

Fig. 5 For each of the three areas North America (top left), Europe (top right), and the tropics (bottom), the plots show the total perceived wind forecast error for the vector wind, and the calculated observation error and prediction error as indicated in the legend. The calculations are based on 6-hour forecasts for the first quarter of 1984 with the ECMWF system; the unit is $\mathrm{m} / \mathrm{s}$. In all three areas the prediction error and observation error are comparable in the troposphere. (from Hollingsworth et al. 1986). 
system.

\section{b) Long term Data monitoring}

The system of near real-time data monitoring at ECMWF has developed over the years and is very effective in detecting errors in the data, and weaknesses in the assimilation system. However it has been possible to refine the system even further by considering monthly or longer term statistics on the diferences between the observations on the one hand and the background and analysed fields on the other.

Hollingsworth et al. (1986) demonstrated that monthly or longer term statistics on the treatment of data in the assimilation can reveal weaknesses both in the data and in the assimilation system itself. Calculations to partition short range forecast errors, as perceived by rawinsondes, into spatially correlated prediction errors and spatially uncorrelated observation errors (which include sampling errors) indicated that the two components of perceived forecast error are of comparable magnitude when good quality data is regularly available, Fig. 5 .
There are three components of the assimilation system: the 6-hour forecast, the analysis, and the initialisation. If the forecast error and observation error are comparable in data rich areas, then most of the atmospheric evolution from one analysis time to the next must have been accomplished by the forecast step. Hollingsworth et al. (1986) demonstrated this by comparing the RMS changes made by each of the three steps in the assimilation from $0600 \mathrm{GMT}$ to $1200 \mathrm{GMT}$ in October 1983. For $500 \mathrm{hPa}$ geopotential they found the northern hemispheric rms changes to be $21.6 \mathrm{~m}$ for the forecast, $12.5 \mathrm{~m}$ for the analysis and $6.5 \mathrm{~m}$ for the initialisation. This state of affairs has come about because of many developments in the techniques of analysis assimilation and forecasting. Fig. 6 compares the perceived 6-hour forecast errors over North America in the fall of 1974 (Hollett, 1975) and in summer and winter 1983 (Hollingsworth et al., 1986), which shows clearly the extent of the improvement in technique over the last decade.

The reliable quality of the analyses and 6 -hour forecasts is exploited in a number of a) HEIGHT / NORTH AMERICA

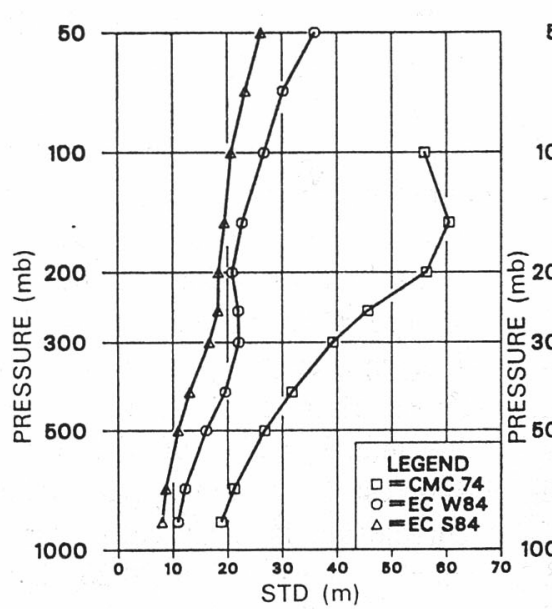

b) WIND / NORTH AMERICA

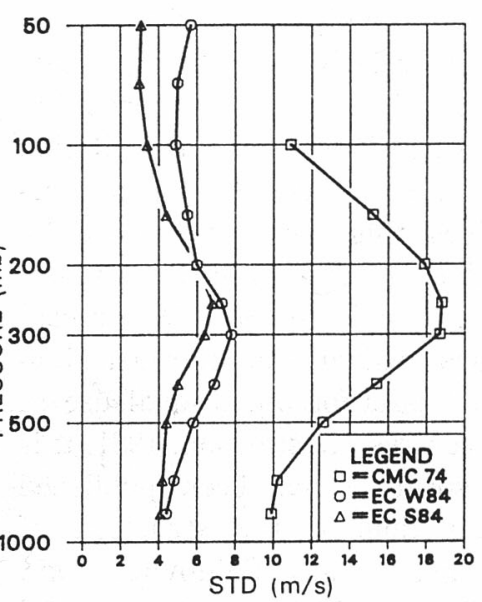

Fig. 6 The plots show the perceived forecast errors for height (left, unit $\mathrm{m}$ ) and wind (right, unit $\mathrm{m} / \mathrm{s}$ ) for verifications against North American radiosonde data. The curves labelled CMC74 are from Hollett (1975), and the ECMWF forecasts for Jan-Mar 1984 and Jun-Aug 1984 are indicated by EC W84 and EC S84 respectively (from Hollingsworth et al. 1986). The substantial reductions in short range forecast errors indicate the strides that have been made in data assimilation over the last decade. 
ways. Delsol (1985) has presented monthly means of the bias and standard deviations of the differences between the forecasts and retrieved thickness temperatures from the TOVS systems on the NOAA satellites. These have been helpful in identifying the areal distribution of biases, particularly at low levels, in the retrieved temperatures.

Delsol has also demonstrated that near midlatitude jet-streams there are substantial biases in the differences between cloud track wind reports and the first-guess, while the equivalent biases for radiosondes are much smaller. Pierrard (1985) and Kallberg and Delsol (1986) have used rawinsonde, aircraft, and cloudtrack winds in collocation studies which show clearly that there are indeed substantial speeddependent biases in the operational Cloud Track wind data. Several possible solutions are under consideration. For the re-processing of the FGGE data it was decided to use the collocation studies to calibrate some of the cloud track wind data near the jet-streams of the Southern Hemisphere.

\section{The statistical structure of short range forecast errors}

As emphasised in many papers on $\mathrm{O} / \mathrm{I}$, the statistics of the first-guess error play a central role in the $\mathrm{O} / \mathrm{I}$ analysis algorithm, both in determining the relative weights of firstguess and observation, and in determining the response characteristics. The assimilation uses the observations to correct forecast errors, and it does this most effectively by spreading out the information in the observations to the extent indicated by the forecast error correlations. Given that the $\mathrm{O} / \mathrm{I}$ algorithm has been adopted for operational use at many different centres (Gustafsson, 1981) it is surprising that so little has been published on the determination of the structure of forecast errors (Hollett, 1975; Balgovind et al., 1983; HL/LH, 1986; Thiebaux, Shantz and Mitchell, 1986), as distinct from the statistical structure of departures from climatology.

\subsection{Horizontal structure functions for mass and wind}

The papers by HL/LH used the formula- tion of the last section to study short range (6-hour) forecast errors in the ECMWF assimilation, using North American rawinsonde data for verification. The first step was to partition the perceived forecast error into horizontally correlated prediction error and hrizontally uncorrelated observation error. The technique used, the extrapolation of horizontal lag correlations to zero lag, was introduced by Drozdov and Shepelevskii (1946), and has been widely used since then. The horizontally correlated prediction errors in height and wind are each partitioned into a large scale part, which is essentially constant over a large area, and a synoptic scale part. The synoptic scale wind components are partitioned into a rotational part and a divergent part. The Bessel expansions used for the extrapolation were truncated at a scale corresponding to planetary wave-number 68 (i.e. a horizontal wavelength of about $600 \mathrm{~km}$ ). Forecast error on shorter scales was regarded as sampling error and therefore included in the estimate of observation error.

Forecast error and radiosonde observational error are of comparable magnitude for both height and wind over land areas of the Northern Hemisphere, and indeed in the Tropics, in those areas where good quality data is available, Fig. 5 (HL/LH, Hollingsworth et al. 1986). The mid-latitude wind forecast errors on synoptic scales are mainly non-divergent and geostrophic. The Rossby number of the forecast errors is about 0.4 , and the geostrophic coupling coefficient between geopotential and stream-function is about 0.9 . The spectra of the correlations are well behaved, with spectra being flatter, or whiter, at lower levels than at higher. This indicates that the length scales of the forecast error are shortest in the lower troposphere. The $\langle\psi, \chi\rangle$ correlation is practically zero. There is a marked correlation (0.25) between $\phi$ and $\chi$, corresponding to excessive convergence in the lower troposphere when a low is forecast too deep. A similar feature is noted in the behaviour of the NMC regional model (N.A. Phillips, pers. comm.)

A substantial part of the forecast error in both height and wind is on horizontal scales 
larger than planetary wave no. 8; we shall call this the large scale error. The large scale wind error changes phase between the lower stratosphere and the lower troposphere. The large scale geopotential error is nearly barotropic, Fig. 7b. A study of the height and temperature errors of the Canadian assimilation (Thiebaux, Shantz and Mitchell, 1986) shows indications of a similar large scale error. These authors found that the horizontal correlation length was much shorter for temperature than for geopotential, and that part of the difference could be explained by a large scale error in the height field.

\subsection{Vertical structure functions for mass and wind}

Provided the assumptions of homogeneity and isotropy are valid for vertical differences of height or wind, the kinematic equations, Eq. 5.1, are as valid for the vertical differences as they are for the height and wind at a single level. HL/LH used this result to determine the vertical correlation matrices for forecast error and observation error in both height and wind.

Both Bergman (1979) and Lorenc (1981) formulated the 3-dimensional structure function for height and wind in separable form as a product of a function describing the horizontal dependence and a function (continuous or discrete) describing the vertical dependence. With this formulation, the maintenance of the geostrophic constraint required that the same vertical function be used for height and wind.

Figure 7, from HL/LH, shows plots of the vertical correlations of the synoptic scale and large scale height errors, and of the wind errors. The height and wind data have very different vertical dependences, which is clear evidence for non-separability. Moreover the vertical correlation structure of the individual terms in the expansions for the synoptic scale height field showed marked variability from one term to the next, which was also inconsistent with the presumption of separability.

An explanation of these results was suggested by Phillips (1986) who modelled the forecast errors in a data rich area as an ensemble of slow modes with random phases. Phillips assumed that the ensemble had a white noise spectrum in the sum of potential and kinetic energy. Balgovind et al. (1982) had earlier proposed a model which assumed that forecast errors had a white noise spectrum in potential vorticity; neither spectrum quite fits the empirical results. Phillips has shown that if his formulation takes account of the vertical and horizontal resolution of the ECMWF model, he can reproduce many important features of the results of $\mathrm{HL} / \mathrm{LH}$, such as the overall variation in the forecast error amplitudes, and the sharper vertical correlation functions for wind as compared with height. Most operational systems use the separability assumption that a three dimensional correlation may be represented as a product of two functions-one describing the horizontal and the other the vertical dependence. Geostrophy then requires that the vertical correlations for height and wind be the same. Phillips' model is inherently nonseparable, with a different vertical dependence for each horizontal component of the turbulent ensemble; Fig. 7 is clear evidence for nonseparability in the ECMWF assimilation. As a further result of the non-separability, Phillips' model of forecast error predicted that the horizontal length scale for temperature should be much shorter than the length scale for geopotential. This feature was found in the Canadian results and is confirmed in unpublished ECMWF results.

Altogether we now have substantial theoretical and empirical evidence for the existence of marked non-separability in the forecast error statistics of operational data assimilations. One must now consider seriously the need to use a non-separable correlation model to make an optimal use of data sources like rawinsondes and satellite thicknesses. This might be expensive in computer time, should it prove necessary, but the cost will be negligible compared to the cost of the observations.

There are important features of the HL/LH results which are not explained by the simple model of Phillips. Notable amongst these is the maximum in forecast error near the tro- 
a)

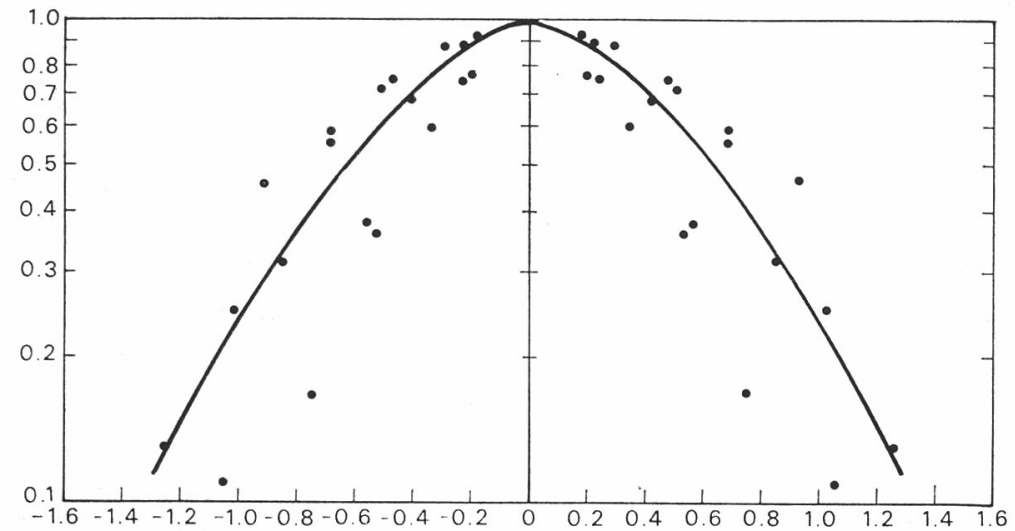

b)

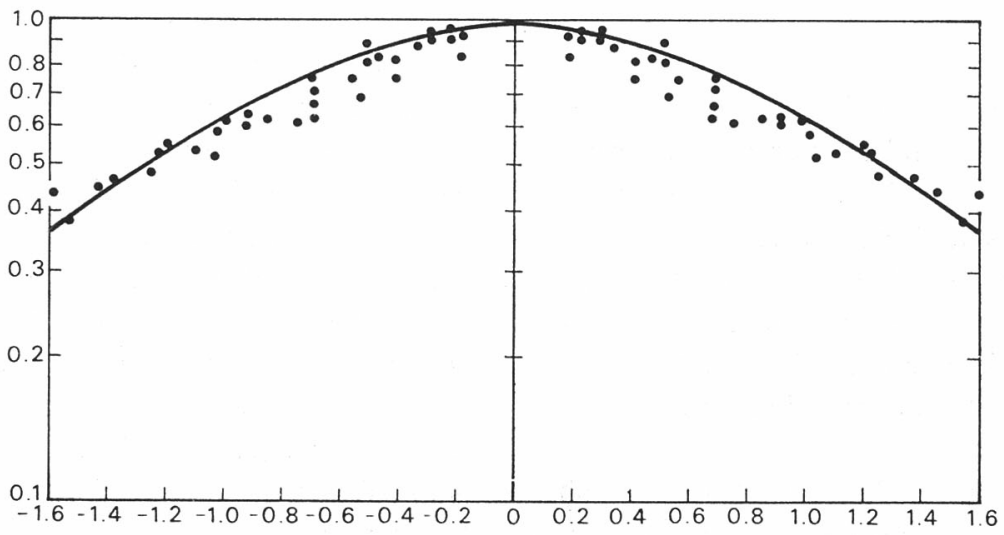

c)

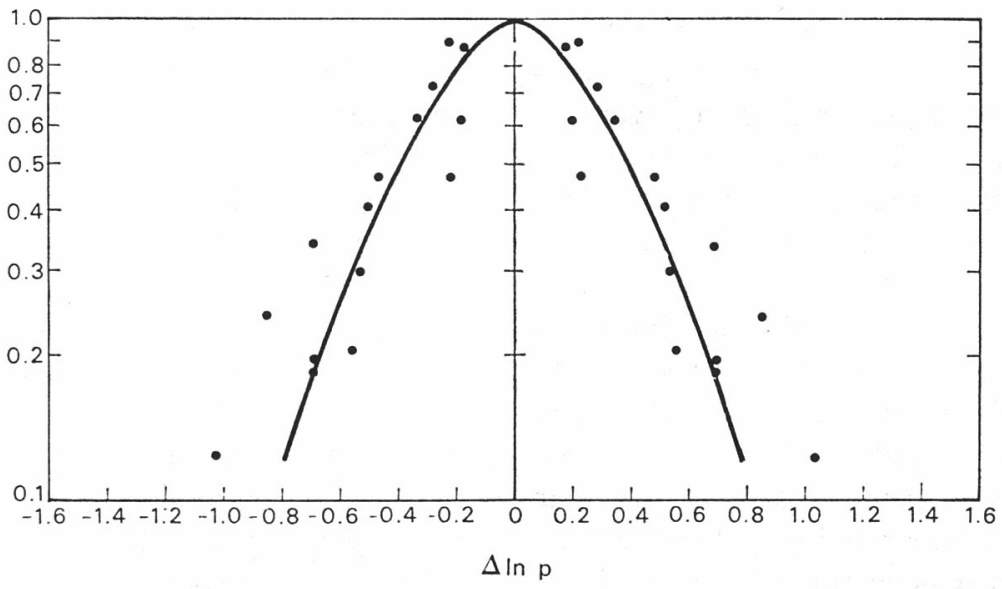

Fig. 7 The plots show the vertical correlation (on a log scale) of a) synoptic scale height forecast errors, b) large scale height forecast errors, c) synoptic scale wind errors, as functions of the difference in the logarithms of the pressures for standard levels below $150 \mathrm{mb}$, using 6hour ECMWF forecasts verified against radiosondes over North America for the first quarter of 1983. If the errors were separable and geostrophic then the curves a and $c$ would be identical. Panel b shows the nearly barotropic nature of the large scale forecast error in height. (from Hollingsworth and Lönnberg 1986, Lönnberg and Hollingsworth 1986). 


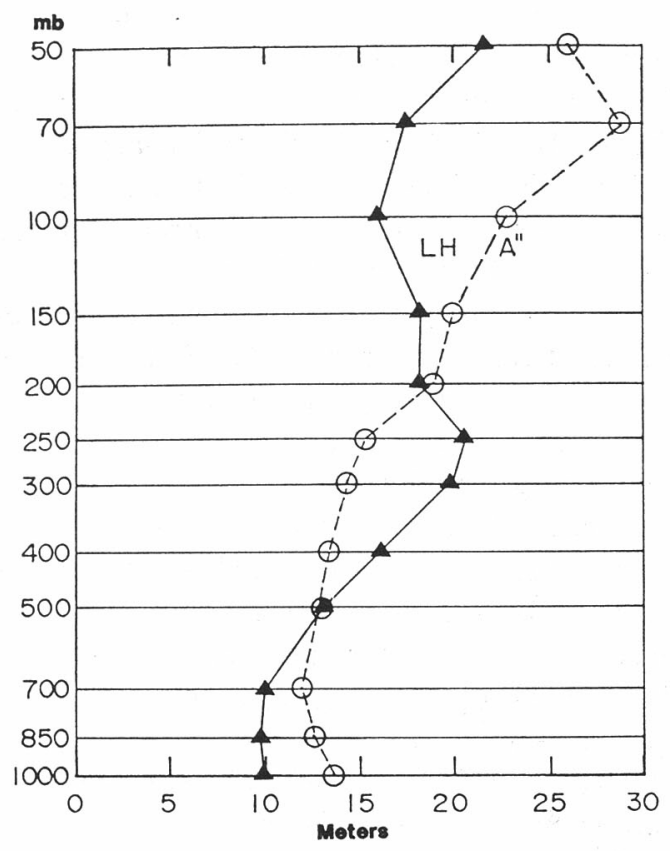

Fig. 8 Distribution of square root of height variance as determined for the ECMWF forecast errors by Lonnberg and Hollingsworth, 1986, $(\mathrm{LH})$, and for an ensemble of idealised slow modes $\left(A^{\prime \prime}\right)$. The overall similarity of the curves indicates that the idealised ensemble can reproduce some properties of the empirical results. (from Phillips 1986).

popause, both in height, Fig. 8, and wind (not shown). Propagation of error from the data-poor oceans may be an important factor, but modelling problems near the troposphere could also contribute. The shape of the spectra found in HL/LH's results also need explanation; they vary from about $k^{-1.5}$ at low levels to about $k^{-3}$ at upper levels. The large scale errors also need explanation; there is some indication that they may be partly tidal in origin. Thiebaux et al. found no evidence for tidal effects in their calculations. Accurate treatment of the tides in an assimilation requires a good diurnal cycle in the forecast model, Unden (1984), and a suitable initialisation condition, Wergen, (1986).

Balgovind et al. (1983) proposed a theoretical beta-plane model for forecast error and found a marked latitudinal variation in the scale of mass-field forecast errors, in accordance with their assimilation results. The scale variation was larger than simple $f$-plane theory would indicate (Phillips, 1986). Thiebaux et al. (1986) found a similar empirical result. HL/LH did not pursue this line of investigation because of known problems in their system in large scale analysis in the tropics, in modelling of the tropics, and in assimilation of tides. Given recent progress in the assimilation of tidal data (Wergen, 1986), and in the analysis and forecasting of large scale fields in the tropics (cf para 8.3), a re-examination of the latitudinal dependence of forecast errors would be interesting.

\subsection{Significance of forecast error structure in data rich areas}

It has been argued since Gandin (1963) that the structure functions have little effect on analyses in data rich areas, and are only important in data sparse areas; the comment was made in the context of analysis using climatology as a background field. The results of Seaman and Hutchinson (1985) however suggest that the correlation functions are indeed important in data rich areas. We have some results which support their conclusion, rather than Gandin's, in current operational work.

One of the major changes introduced in the ECMWF assimilation by Shaw et al. $(1984,1987)$ was an increase in the vertical and horizontal resolution of the analysis, by using sharper structure functions; the changes were based on the results of $\mathrm{HL} / \mathrm{LH}$. There were other important changes as well, affecting data selection and quality control. The impact of these changes has been shown in the verifications of the short range wind forecasts over North America in the winter of 83/84 (after the introduction of the T63 spectral model) and in the winter of $84 / 85$ after the introduction of the analysis changes (Fig. 2). The impact of the analysis changes has been very positive. One may conclude that the correlation functions do indeed matter in the relatively datarich area of North America.

When using climatology as a background field (Gandin 1963) in an area such as North America or Europe, the observations are very close to each other relative to the width of the auto-correlation of background error. The 
larger scales find a much better response than the smaller scales: as a result the details of the correlation structure do not matter provided they represent the main broad scale features well.

When one is analysing forecast errors in an area such as North America, the scales of interest are much shorter than they are when analysing climatological fluctuations. The data density relative to the correlation length of the background field is correspondingly lower and as a result the importance of the statistics of the background field increases.

\subsection{One-point correlations, anisotropy and flow dependence}

A common criticism of operational $\mathrm{O} / \mathrm{I}$ systems is that the correlations used are isotropic and make no attempt to use functions elongated along the flow direction. Some of this criticism is based on a mis-understanding of the fact that an operational system analyses corrections to the forecast, rather than the entire spectrum of atmospheric fluctuations. If the anisotropies have been captured by the first-guess, then the first-guess errors may not be anisotropic, and the analysis problem is therefore simplified.

Holopainen, (1978), Hoskins, James and White (1983), use the tensor of one-point velocity covariances, or Reynolds stress tensor, to summarise important aspects of the anisotropy of a two-dimensional velocity field. They write the velocity correlation tensor in the form

$$
\left(\begin{array}{ll}
\overline{u^{\prime} u^{\prime}} & \overline{u^{\prime} v^{\prime}} \\
\overline{u^{\prime} v^{\prime}} & \overline{v^{\prime} v^{\prime}}
\end{array}\right)=\left(\begin{array}{ll}
K & 0 \\
0 & K
\end{array}\right)+\left(\begin{array}{cc}
M & N \\
N & -M
\end{array}\right)
$$

where

$$
\begin{aligned}
& K=\left(\overline{u^{\prime} u^{\prime}}+\overline{v^{\prime} v^{\prime}}\right) / 2 \\
& M=\left(\overline{u^{\prime} u^{\prime}}-\overline{v^{\prime} v^{\prime}}\right) / 2 \\
& N=\overline{u^{\prime} v^{\prime}}
\end{aligned}
$$

The prime denotes the departure from the ensemble mean and the bar denotes ensemble mean. The principal axes of the Reynold's stress tensor have magnitudes $K \pm\left(M^{2}+N^{2}\right)^{1 / 2}$, and the major axis has an orientation $1 / 2$ $\tan ^{-1}(N / M)$ to the $x$-axis. In the northern hemisphere an orientation from South-West to North-East indicates a poleward momentum flux. The 'anisotropy' vector $V$, with magnitude $\left(M^{2}+N^{2}\right)^{1 / 2}$ and orientation along the major axis, gives a convenient summary of the main properties of the anisotropy for the one-point velocity correlations.

We now apply these concepts to the anisotropies of forecast error fields. Fig. 9a shows plots of the anisotropy vector for the $250 \mathrm{hPa}$ wind forecast errors at the North American radiosondes for winter $1984 / 85$. The vectors are oriented mainly North-South near the West Coast, and are oriented mainly EastWest in the East. The magnitude of the anisotropy vectors for the forecast errors is about $1 / 25$ of the atmospheric magnitude. Fig. $9 \mathrm{~b}$ shows the ratio $\alpha=\left(M^{2}+N^{2}\right)^{1 / 2} / K$, which gives a non-dimensional measure of the significance of the anisotropy in the wind forecast error. Typical values are $0.2-0.3$ $K$, the denominator of $\alpha$, contains contributions from observation error and forecast error since $K$ is the energy of the measured forecast error. If the observational error is isotropic, and is comparable with the prediction error, the true anisotropy of the forecast errors may therefore be of order 0.5 , which is comparable with values for atmospheric fluctuations. Thus anisotropy may be as significant a feature of the forecast errors as it is of the observed fields. Preliminary investigations indicate that the residual anisotropy after the analysis step is rather small in the observation minus analysis differences.

The anisotropy calculations just discussed can only document the existence of components of the correlation structure with azimuthal wave number $m=2$, if the forecast errors are homogeneous. As discussed by $\mathrm{HL} / \mathrm{LH}$ the components with odd parity $(m=1,3$, etc.) occur in cross-correlations and may be important in describing the baroclinic phase tilts in the forecast errors. Evidence for the existence of these components is forth-coming in certain types of flow (A.M. Jørgensen 1986, pers comm).

A full investigation of the need for anisotropy in the forecast error correlation model will need to consider the balance between the 


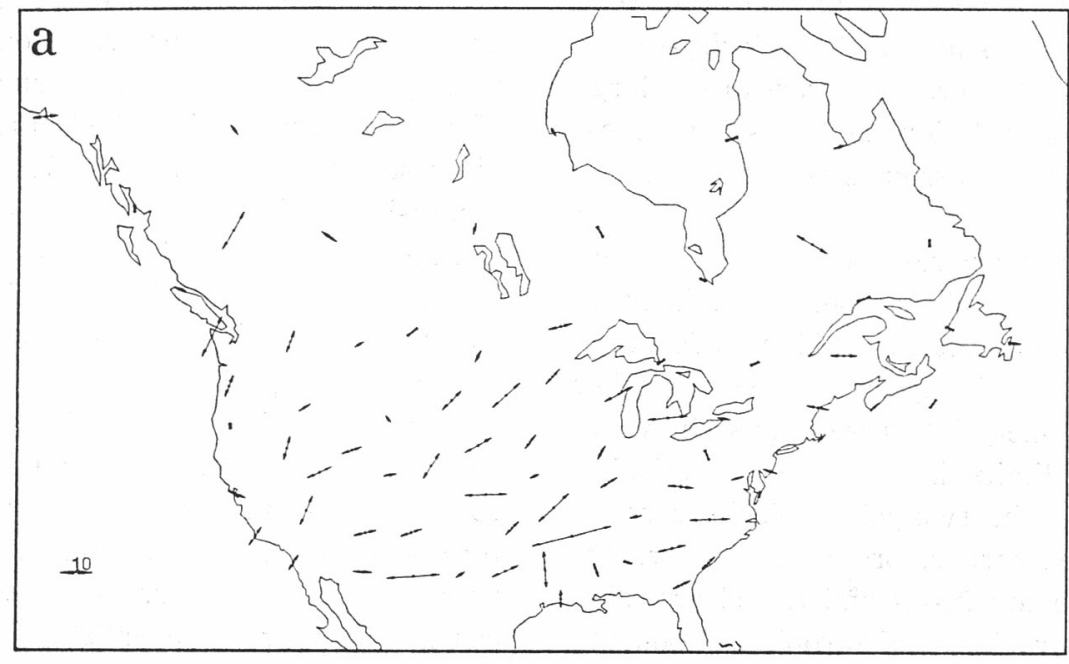

ANISOTROPY(OB-FG) VECTOR WIND 250 HPA

DECEMEER 84 - FEBRUARY $85 \quad 12$ GMT

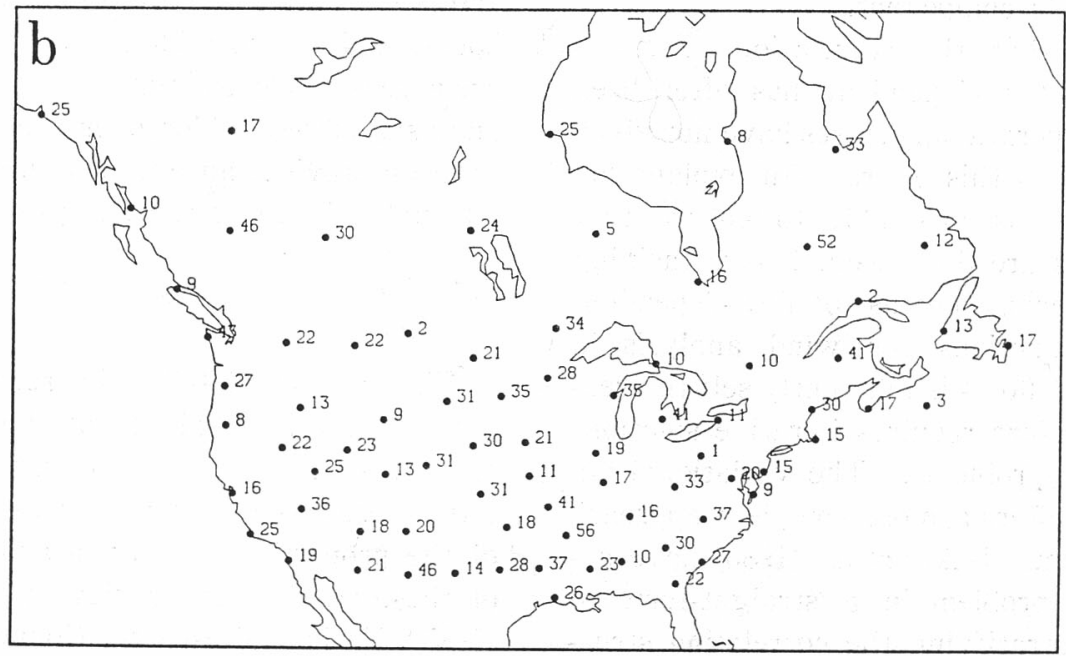

Fig. 9 a) The anisotropy vector [magnitude $\left(M^{2}+N^{2}\right)^{1 / 2}$, and angle $1 / 2 \tan ^{-1}$ $(N / M)$ from latitude circle] of ECMWF 6-hour wind forecast errors verified against North American radiosondes for 1200 UT in December 1984-February 1985; the unit is $(\mathrm{m} / \mathrm{s})^{2}$. The coherent patterns and the magnitudes of order $25(\mathrm{~m} / \mathrm{s})^{2}$, indicate that there is substantial anisotropy in the forecast errors. b) The ratio $\alpha=\left(M^{2}+N^{2}\right)^{1 / 2} / K$ for the vectors shown in a), plotted as a percentage. If half of $K$ is observational error, then these numbers may be doubled, indicating that anisotropy is as significant for the forecast errors as it is for the atmospheric fields.

mass and wind field. A convenient survey of the role of anisotropy and ageostrophy in the mass-wind coupling of the forecast errors is provided by the vector of one-point correlations $\left(\overline{\phi^{\prime} u^{\prime}}, \overline{\phi^{\prime} v^{\prime}}\right)$. For atmospheric fluctua- tions this quantity shows the mainly nondivergent pattern expected from quasi-geostrophic flow (Holopainen, 1978), with indications of the smaller terms arising from correlations of $\phi$ with the divergent wind 
(Hoskins and Sardeshmukh, 1986). Calculations of this vector for the forecast errors shows that it is noisier than the anisotropy vectors. At some stations the velocity-height correlations in the forecast errors are as large as 0.5. The correlation model used at most operational centres assumes that this correlation is zero.

These preliminary results suggest that there may be a need to take account of anisotropy and divergence in the correlation models used for analysis, but the question needs further study. The one-point correlations shown in Fig. 9 are the limits, in the case of vanishing spatial lag, of the two-point velocity correlations discussed earlier, provided the forecast errors are homogeneous and the observation errors are isotropic. Anisotropic components of the forecast errors can be incorporated in a correlation model using the same formalism as the isotropic component.

The view that the correlation functions ought to be flow-dependent has often been expressed. Operational centres have not given high priority to this effort. An explanation may be that it is profitable to ensure that simple things are done well, before tackling difficult problems. The use of flow-dependent correlation functions for wind analysis in highly curved flows is inherently self-referential, and therefore requires iterative solutions to non-linear problems. The variational/adjoint method offers a more complete approach to the problem. Baker et al. (1986) have approached the problem in a straight-forward fashion by stratifying the correlation structures of analysis increments according to the background vorticity, but using isotropic representations for each of the vorticity categories. The results seemed to indicate modest improvements.

\section{The magnitude and significance of analysis errors}

The analysis error field is, by definition, the difference between the analysed field and the true field. Since the true field can never be known exactly, the analysis error field can never be known exactly. It can nevertheless be estimated, if only approximately.
Many techniques have been used to assess the magnitude and significance of analysis errors. Forecast error was discussed earlier as a proxy for analysis error. Comparisons of independent analyses, and a-priori estimation of analysis error using the $\mathrm{O} / \mathrm{I}$ technique, are discussed in sections 7.1 and 7.2 respectively. Analysis verification using two-point correlations of observation minus analysis differences is discussed in para 7.3; analysis verification using one-point correlations has been touched on already in para 6.4 .

For those with access to a good forecast model, the best estimate of analysis accuracy comes from verification of short rangeforecasts based on the analyses. The advantage is that the errors of the verifying observations are independent of the forecast errors, if the observations are unbiased. With a 2-day doubling time, mid-latitude analysis errors should amplify by less than 20\% in 12 hours, and so the mid-latitude forecast errors are a reasonable estimate of the mid-latitude analysis errors. Moreover the forecast verification provides an excellent measure of the internal balance of the analysis.

\subsection{Comparison of operational and FGGE analyses}

With the availability in recent years of many different analyses of the FGGE data, and, of several different operational analysis sets, there have been numerous comparisons of the properties of different analyses. Many of these studies are reviewed in Miyakoda (1985), National Research Council (1985) and WMO (1985b). These investigations have used a variety of methods to compare the analyses. Some have compared the energetics of the analyses, some have examined the intensity of the diabatic processes implied by the analyses; other have studied the accuracy of forecasts made from different analyses, and yet others have considered the internal balance of the analyses. Implicit in all these studies has been an attempt to estimate the accuracy and consistency of the analyses by establishing the range of uncertainty in the analyses (WMO, 1985b).

The simplest comparison method is a com- 
putation of the rms differences between independent analyses of the same observations (Arpe (1980), Rinne and Jarvenoja (1983), Lau (1984) and many other authors). If the analyses are assumed to be of equivalent accuracy, and if their errors can be assumed to be uncorrelated, then the variance of the analysis differences may be attributed equally to errors in both analyses. This method has been used extensively by Rinne and Frisk (1979) and by Rinne and Jarvenoja (1983) to estimate analysis errors of long series of operational analyses, and of analyses of the FGGE data; their results demonstrate the high accuracy of the FGGE analyses.

In addition, Rinne and Jarvenoja (1984) developed an alternative technique for estimating analysis error. They projected sets of $500 \mathrm{hPa}$ height analyses onto empirical orthogonal functions (EOF's). Using time series analysis methods, they then developed optimal linear predictors for the evolution of the time series of the EOF coefficients. These methods provide an estimate of the noise in the time series of coefficients, which they attribute to analysis error. The results they found with this method had many important similarities with the results they obtained from computations of the rms differences.

\subsection{The O/I estimate of analysis error}

The $\mathrm{O} / \mathrm{I}$ analysis method provides an estimate of the second moment of the analysis error (Gandin, 1965). Seaman $(1977,1983)$ has shown that the analysis error can be calculated in a similar way for any analysis method which defines an analysis increment to a background field by a linear combination of the observations. The estimated analysis error is used in quality control of data (Lorenc 1981), in estimating the accuracy of shortrange forecasts made from the analyses (Lorenc, 1981; Ghil et al., 1981), and therefore in deciding on the relative weights to be given to observations and the back-ground field. The estimates appear to be rather reasonable in data sparse regions when compared with the independent estimates of Rinne and Jarvenoja (1984).

However the estimated analysis error ap- pears to be over-optimistic if a great deal of data is used in the estimation of analysis error in a data rich area. Experiments have shown that if height data from a single radiosonde in Central Europe is withheld from the analysis, then the estimated error in 500 $\mathrm{hPa}$ height is of order $2 \mathrm{~m}$, when the actual analysis error is between 5 and $8 \mathrm{~m}$, due allowance being made for observation error (Lönnberg and Shaw, 1984). As discussed by Seaman (1977), Franke (1983) and Lorenc (1986), the $\mathrm{O} / \mathrm{I}$ estimate of analysis error is probably much less reliable than the $\mathrm{O} / \mathrm{I}$ analysis in these circumstances. The main reason is that the estimate makes no allowance for inevitable errors in its own statistics, and the estimate tends to zero as the number of data becomes large. Since the estimated analysis error is a monotonically decreasing function of the number of data used, it will tend to give unrealistically optimistic estimates of analysis error if large valumes of data are used.

\subsection{Verification of analyses using two-point correlations}

Computations of rms differences between analyses and observations are a standard verification tool. At best such comparisons can tell if the data were used in the analyses, but there is little further information. Hollingsworth and Lönnberg (1987) have shown that a study of the spatial correlation structure of the observation minus analysis (Ob-An) differences can yield upper and lower bounds on the magnitudes of analysis errors, and on the spectra of analysis errors, in much the same way as study of the correlation structure of the observation minus forecast $(\mathrm{Ob}-\mathrm{Fg}$ ) differences can quantify the magnitude and spectrum of forecast errors.

\section{a) The theoretical covariance of observation minus analysis differences}

To provide a theoretical context for the discussion of the observation-minus-analysis (Ob-An) differences, Hollingsworth and Lönnberg (1987) show that in the case of a complete $\mathrm{O} / \mathrm{I}$ analysis

$$
A=P-P \cdot(P+D)^{-1} P
$$


where $\boldsymbol{A}$ represents the covariance matrix of the analysis errors at the observation points. From this result one may derive an expression for the covariance of the difference between the observed and analysed values

$$
\operatorname{Cov}\left\langle(d-a) \cdot(d-a)^{*}\right\rangle=\boldsymbol{D}-\boldsymbol{A}
$$

If the observations were not used in the analyses the convariance of the Ob-An differences would be

$$
D+A^{\prime}
$$

where $\boldsymbol{A}^{\prime}$ is the analysis error covariance if the observations in question are not used. If the observations were used but the analyses were less than ideal one would expect to find that

$$
\operatorname{Cov}\left\langle(d-a) \cdot(d-a)^{*}\right\rangle=\boldsymbol{D}+\boldsymbol{A}-2 \boldsymbol{E}_{0}^{*} \cdot \boldsymbol{R}_{o / a} \cdot \boldsymbol{E}_{a}
$$

where $\boldsymbol{R}_{0 / a}$ is the correlation matrix of observation error and analysis error at the obervation points, and $\boldsymbol{E}_{0}, \boldsymbol{E}_{a}$ represent the vectors of rms observation error and analysis error at the observation points. Consistency of this result with Eg. 7.2 requires that in the complete $\mathrm{O} / \mathrm{I}$ analysis :

$$
\boldsymbol{E}_{0}^{*} \cdot \boldsymbol{R}_{0 / a} \cdot \boldsymbol{E}_{a}=\boldsymbol{E}_{0}^{*} \cdot \boldsymbol{R}_{a / a} \cdot \boldsymbol{E}_{a}(=\boldsymbol{A})
$$

where $R_{a / a}$ is the auto-correlation matrix for analysis error. Since $\boldsymbol{R}_{a / a}$ tends to the identity matrix $I$ at zero separation, Eq. 7.3 suggests that the spatial correlation of Ob-An differences should be negative at short separations, provided observation errors are spatially uncorrelated and sampling errors are negligible. We shall now demonstrate this in some simple calculations.

\section{b) Two-point correlations of observation- analysis differences}

Given a set of analyses and observations, the spatial variation of the covariance of the Ob-An differences at points 1 and 2 may be written

$$
\begin{aligned}
& \operatorname{Cov}\left[(o b-a n)_{1},(o b-a n)_{2}\right] \\
& \quad=\varepsilon_{o}^{2} \delta_{12}-2 \varepsilon_{o} \varepsilon_{a} \rho_{a / 0}(r)+\varepsilon_{a}^{2} \rho_{a / a}(r)
\end{aligned}
$$

where $r$ is the separation between points 1 and 2 $a b=$ observation error, with assumed zero mean and no spatial correlation, $a n=$ analysis error, with assumed zero mean,

$\varepsilon_{0}=r m s(o b)$,

$\varepsilon_{a}=r m s(a n)$,

$\rho_{a / 0}(r)=$ correlation of observation and analysis error, and

$\rho_{a / a}(r)=$ auto-correlation of analysis error.

$\delta_{12}=$ Kronecker delta

Considering only the isotropic component of the Ob-An covariance statistics, we may define the function $b(r)$ by

$$
b(r)=-\left[2 \varepsilon_{0} \varepsilon_{a} \rho_{a / 0}(r)-\varepsilon_{a}^{2} \rho_{a / a}(r)\right]
$$

This function of station separation can be fitted in a least squares sense by a truncated series of Bessel functions of zero order. The truncated series expansion may be extrapolated to the origin to give an intercept $b(0)$.

We then have the relations

$$
\varepsilon_{o}^{2}=\left.[r m s(o b-a n)]^{2}\right|_{r=0}-b(0)
$$

and

$$
\varepsilon_{a}^{2}-2 \varepsilon_{0} \varepsilon_{a} \rho_{a / 0}(0)-b(0)=0
$$

Defining $\varepsilon=\varepsilon_{a} / \varepsilon_{o}$ and $b=b(0) / \varepsilon_{o}^{2}$, where $\varepsilon_{0}$ is normalised by the first guess error, Hollingsworth and Lönnberg (1987) show that if the intercept $b$ is negative then

$$
1-(1-|b|)^{1 / 2}<\varepsilon<1 /\left(1+\varepsilon_{o}^{2}\right)^{1 / 2}
$$

while if the intercept is positive then

$$
(b)^{1 / 2}<\varepsilon<1 .
$$

These bounds on the analysis error at observation points are instructive. The analysis error at observation points is smaller when the intercept is negative than when it is positive. The closer to zero the negative intercept, the smaller the analysis error at the observation points. Hollingsworth and Lönnberg (1987) use these results to estimate the analysis error at observation points. They also show that equivalent bounds on the spectrum of analysis error may be derived. As an example of their results, Fig. 10a shows the spatial correlation of their operational $500 \mathrm{hPa} \mathrm{Ob}-\mathrm{An}$ differences in the height 
ANALYSIS ERROR $500 \mathrm{HPA}$

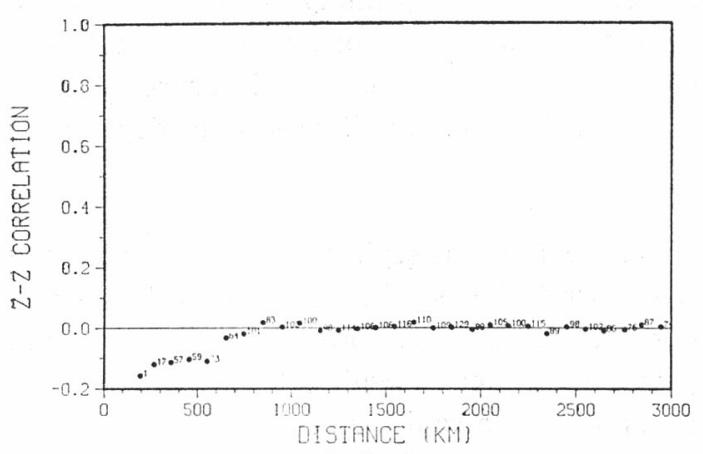

(a)

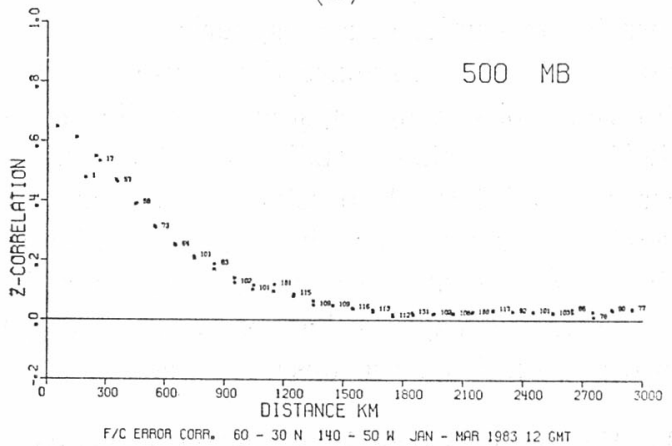

(b)

Fig. 10 a) The spatial correlation of Ob-An differences in $500 \mathrm{mb}$ height for $1200 \mathrm{UT}$ radiosonde data over North America in December 1984-February 1985. The empirical data, averaged over bins of $100 \mathrm{~km}$, are shown by squares, and a least squares fit to the data is shown by ' $x$ '. The figures indicate the number of station pairs in each bin. Each pair had a minimum of 60 reports for the calculation. The negative intercept can be used to put an upper bound on the analysis error at the grid points from Hollingsworth and Lönnberg, 1987 . b) As a) for the Ob-Fg differences for the first quarter of 1983. (from Lonnberg and Hollingsworth 1986).

field. The negative intercept at the origin is quite different from what one sees with $\mathrm{Ob}-\mathrm{Fg}$ differences (Fig. 10b). The results in Fig. 10a suggest that the analysis error variance at observation points is about one quarter of the observation error variance, at most. Similar calculations can be made for the thickness, for the wind, and for the wind shear.

\section{c) Diagnosing the efficiency of an analysis}

Apart from their value in providing bounds on analysis error, the spatial correlations of

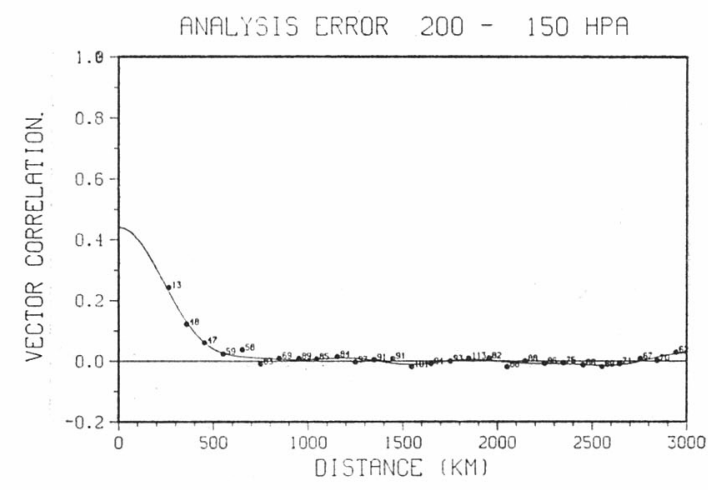

Fig. 11 'The vector correlation of Ob-An differences for the wind shear between $150 \mathrm{mb}$ and $200 \mathrm{mb}$ for $1200 \mathrm{UT}$ radiosonde data over North America in the first quarter of 1983. The empirical data, averaged over bins of $100 \mathrm{~km}$, are shown by squares, and a least squares fit to the data is shown by ' $x$ '. The figures indicate the number of station pairs in each bin. Each pair had a minimum of 60 reports. The positive intercept indicates that the data has not been fully used. The positive intercept can be used to put a lower bound on the analysis error at the grid points fom Hollingsworth and Lönnberg, 1987.

Ob-An differences provide a quick and powerful diagnostic of the efficiency of an analysis system. As an example consider Fig. 11 which shows the correlation of the Ob-An differences for the vertical wind shear between 200 and $150 \mathrm{hPa}$ over North America in Jan.-Mar. 1983. The large positive correlations at separations of $300-700 \mathrm{~km}$ indicate that the analysis algorithm failed to extract this information from the observations, (Hollingsworth and Lönnberg, 1987). This figure is unambiguous evidence that the analysis at the time was not drawing effectively for the vertical wind shear information ( HL/LH; Shaw et al., 1984). The latter paper documents an extensive set of modifications to the system which were implemented in May 1984. Fig. 12 shows the same results as Fig. 11 for the first winter after the implementation. There has clearly been a considerable improvement in the vertical resolution of the wind analysis near the tropopause, and there is still room for further improvement.

These methods enable one to determine if a three-dimensional analysis has used to the fullest extent the data available to it. They 


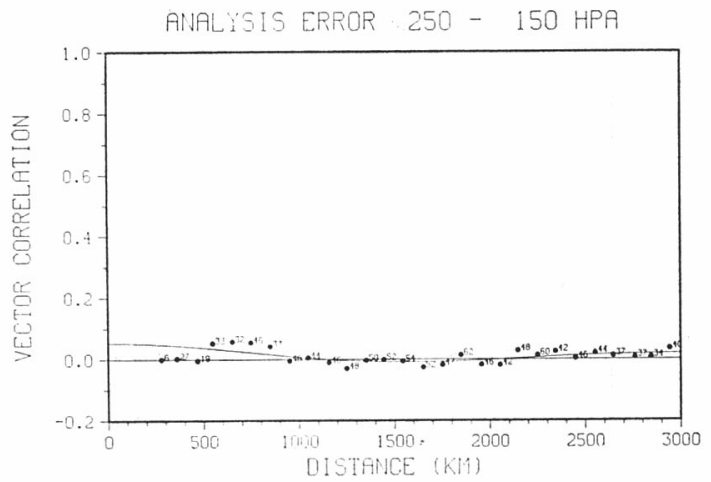

Fig. 12 The vector correlation of Ob-An differences for the wind shear between $150 \mathrm{mb}$ and $200 \mathrm{mb}$ for $1200 \mathrm{UT}$ radiosonde data over North America in December 1984-February 1985. The empirical data, averaged over bins of $100 \mathrm{~km}$, are shown by squares, and a least squares fit to the data is shown by ' $x$ '. The figures indicate the number of station pairs in each bin. Each pair had a minimum of 60 reports. The lower values near the origin, in comparison with Fig. 11, indicate that lower bound on the analysis error was much lower in 1984/85 than in early 1983 from Hollingsworth and Lönnberg, 1687.

therefore provide a powerful diagnostic of the efficiency of an analysis by identifying those areas where the analysis is inadequate. We hope that systematic application of these ideas over the next few years will bring us to the stage where we can prove that our analysis algorithm has used all of the available data, to the full. At that point we will need the more explicity four dimensional approach of the adjoint method to make further progress.

\section{d) System dependence of OSE results}

These analysis verification methods provide a useful tool for evaluation and interpretation of Observing System Experiments (OSEs), and of Simulated Observing System Experiments (OSSEs). It has often been found that the results of these experiments are system dependent (Gilchrist, 1982 ; ECMWF, 1984). This leads to difficulties in the interpretation of the results. The verification methods discussed here could be quite valuable in reducing the level of controversy. Experimenters should demonstrate that the analysis system used for the experiments is using all the information available to it from each of the observation system, before beginning their experiments. If this cannot be demonstrated then the outcome of an OSE must be interpreted carefully, as the results will be system dependent.

\section{Discussion}

There is a satisfying symmetry about the way correlation studies can be used to study observation quality, forecast errors and analysis verification. Results of one-point correlations provide a rapid survey and summary of forecast and analysis performance. Two-point correlation studies of atmospheric fluctuations can be used to compare the performance of different observing systems, Wylie et al. (1984). Equivalent studies of the forecast errors provide good correlation functions and good constraints for the analysis. Finally, similar studies of the analyses can determine if the analysis system has extracted all useful information from the observations.

\section{Future research in objective analysis}

We now discuss the areas of objective analysis where research is likely to be concentrated to exploit new technological and scientific possibilities. It has been estimated that machines which are 400 times more powerful than the Cray-1A may be available by 1990 . Today's commercially available machines are about 10 times more powerful than the Cray-1A, ten years after its first introduction. We are therefore in a phase when developments in computers are accelerating rather than otherwise.

There is a steady improvement in telecommunications capacity which makes it possible to disseminate greater volumes of satellite data, much of which is not fully exploited in present operational systems. For example Julian (1985) and Cadet (1983) indicate that cloud top temperatures have information on humidity and divergence which is not exploited in current operational systems. Given that there are now very encouraging prospects for NWP in the Tropics, we can expect substantial developments in exploiting data which is 'available in principle' but not currently used. 
New observational technologies are being introduced, such as the wind sounders which are seen as replacements for the rawinsondes in some areas. It is expected that the Advanced Microwave Sounding System (AMSU), to be flown on operational satellites soon after 1990, will provide as much vertical resolution in cloudy areas as is currently available from the TIROS-N series in clear areas. The potential resolution of this data is of order $80 \mathrm{~km}$ in the horizontal, and $200-300 \mathrm{hPa}$ in the troposphere. In the early 1990s the microwave scatterometers on ESA's ERS-1 satellite, and the U.S. Navy's NROSS satellite will provide near global $50 \mathrm{~km}$ resolution surface wind data. The need to exploit new opportunities will increase the pressure to improve our present methods, as well as raising quite new problems.

With these changes in mind, we may identify four fairly distinct growth areas for research in operational objective analysis in the next few years:

i) High resolution analysis over land (8.1),

ii) Synoptic scale analysis over mid-latitude oceans (8.2)

iii) Synoptic scale analysis over the Tropical Oceans (8.3) and

iv) Quality control of observations (8.4)

Each of the first three areas has different emphasis on the importance of particular types of data, and presents different problems in assimilation of that data. The topic of data quality control is vital for all three areas.

A common theme running through the discussions of each of these areas is the need for more effective use of the time dimension or time history in the analysis. A number of methods are under investigation, some more elaborate than others. They all indicate that a closer meshing of the model and the analysis is possible.

\subsection{Analysis over a wide range of spatial scales}

Short-range forecasts are still the most widely used products of the meteorological services. Since global models with grid-sizes of $50 \mathrm{~km}$ are conceivable by 1990, models covering large regions with much higher resolutions are also conceivable. It has been recognised since the 1950s that objective analysis schemes must be capable of treating a range of spatial scales (Bergthorsson and Doos, 1954; Cress man, 1959).

The method of $\mathrm{O} / \mathrm{I}$ has been used to prepare analyses for the $80 \mathrm{~km}$ Nested Grid Model (Phillips, 1979) at NMC Washington (Dimego, 1985), but the assimilating model which provides the background field is a global model of much lower resolution. Meteorologie Nationale, Paris, runs a regional assimilation cycle where the assimilating model is the 35 $\mathrm{km}$ resolution Peridot model (Pailleux, pers comm.). The method of continuous assimilation has been successfully adapted to run with an operational model having grid spacing of $75 \mathrm{~km}$ at the UK Meteorological Office (Fugard 1985, pers. comm.).

Many questions are still open on what is the best way to approach the problem. If the concept of 'slow' modes is valid in any way at the high end of the spectrum, then Phillips' (1982) results suggest that $\mathrm{O} / \mathrm{I}$ may be the best approach. As implied by Phillips, 'distinguishable' might be a better word than 'slow', since the main requirement for his results on the completeness of $\mathrm{O} / \mathrm{I}$ is that the components of interest be distinguishable in some way from the components which are not of interest. Even if O/I were the optimal approach, there are a number of sub-optimal approaches which could work quite as well in practice, such as continuous assimilation (Fugard, 1984, pers. comm. ; Lyne, 1982 ; DiMego, 1984) or special forms of the Successive Correction Method (SCM) which converge to O/I (Bratseth, 1986; Lorenc, 1986).

\section{a) Scale dependent constraints in $O / I$}

The general formulation for the correlation functions of forecast error discussed in section 6 has many aspects that may be of value in developing an analysis system suitable for application over a wide range of spatial scales, particularly if the dynamical constraints to be imposed on the motion are strongly scale dependent. The spectral representation of the 
correlations permits all the properties of the correlation model to be scale-dependent. It is a simple matter to vary the geostrophic coupling as a function of scale. This would permit the enforcement of a tight geostrophic coupling at synoptic scales, and no geostrophic coupling at very short scales.

Similarly the wind-wind correlation model could permit more divergence at either very large or very small scales than might be desired at synoptic scales, by suitable determinations of the expansion coefficients in the $\langle\chi, \chi\rangle$ correlation. The coupling between the divergent and the rotational winds can be made scale dependent by setting the coefficients of the $\langle\phi, \chi\rangle$ correlations appropriately. Manipulation of the $\langle\chi, \phi\rangle$ correlation might also be useful in particular circumstances, since the correlation is observed to be non-zero.

There is solid empirical and theoretical evidence that the forecast errors are nonseparable. Non-separability and anisotropy in the correlation functions may well become important as resolution is increased. It might be expensive to use a non-separable correlation model in operational work. Such a decision would have to be justified by evidence that the analyses were missing important features of the observations. The methods of analysis verification discussed in the last section would be of value in such a decision.

The spectral formulation requires the specification of a large number of disposable parameters, but it also provides the means of determining those parameters, through spectral decomposition of the forecast errors. The spectral representation can also be useful in deciding if the additional refinements are necessary.

\section{b) Analysis of phenomena having non-linear balance}

As model and analysis resolution is increased, it becomes more and more essential to provide an accurate description of strongly non-linear phenomena such as fronts. If the background forecast has a frontal position error of a few grid-points, linear analysis methods such as $\mathrm{O} / \mathrm{I}$ may be incapable of moving the entire non-linear structure to the correct position. Similar comments may apply to phenomena where the non-linearity involves physical processes in a vital way, as in hurricanes.

Fine mesh analysis for these phenomena will always be faced with a problem of data scarcity. If a synoptician knows that a strong front is to be found in a given area, a few surface reports can often give him an accurate position for the front. To position a front accurately, while providing the forecast model with the full non-linear balance needed for a good forecast, may well require iterative non-linear variational methods using adjoint techniques (Marchuk, 1975; Le Dimet and Talagrand, 1986). This approach may be more productive than trying to develop flowdependent structure functions, where the mathematical formulation is much less clear cut.

\section{c) Analysis near mountains}

The study of the effect of mountains on the atmosphere has been given a substantial impetus by the ALPEX experiment. Excellent reviews of the problem together with summaries of observational, theoretical, and modelling studies may be found in Speranza (1984) and WMO (1986). There have been studies of static fine-mesh analysis of the ALPEX data (Reimer, 1984; Buzzi, 1984; McGinley, 1984), but there is little published material on data assimilation with the ALPEX data with a high-resolution system.

The importance of high resolution models for forecasting near mountains such as the Alps is underlined by the results of Dell'Osso (1984) and McGinley and Goerss (1985). Operational analysis and assimilation systems to support such models will need to consider special features of the flow such as blocking, with consequential in-homogeneity and anisotropy in the forecast errors. Other areas needing consideration will be the structure of the analysis grid, and the data selection strategies, which must also take account of the anisotropies.

\subsection{Analysis over the mid-latitude oceans}

Analysis over the oceans is probably the 
most difficult and important of all analysis problems, because of the scarcity of data over these enormous regions. Apart from the TOVS data, and a few radiosonde reports from ships and islands, all the remaining observation platforms provide information at only one or two levels. Quality control, and analysis, are most difficult over the oceans, because the data is sparse and because the estimates of the first guess error convariances are least reliable there. The distinction between the almost 'climatological' description of forecast error covariances used in many operational systems (Lorenc, 1981) and the current state of the forecast error fields, are most important in such areas. In the view of one eminent authority (N.A. Phillips, pers comm.) "the ability to supply some information on the current forecast error fields is the one facet of the Kalman-Bucy approach that might make the cost of that approach worth considering". Recent work on the variational/ adjoint approach indicate that it, too, can supply such information (Courtier, 1987, pers comm).

\section{a) Use of single level data}

The problem of how to spread out singlelevel data in the vertical is one for which there will never be a fully satisfactory solution. One does not know how much of the information in the observation originates in the barotropic component of the flow and how much in the baroclinic component. One possibility is to treat the information as a deltafunction in the vertical, and not spread it out at all. Barwell and Lorenc (1985) have shown that this is not a good approach.

In the Australian operational system (Bourke et al., 1985) a barotropic interpretation for surface pressure data is adopted. Pressure changes are balanced geostrophically in midlatitudes, and are applied uniformly in the vertical between $1000 \mathrm{hPa}$ and $200 \mathrm{hPa}$. McPherson et al. (1979) and Lorenc (1981) use vertical correlation functions which mean that surface pressure data change the lower tropospheric temperature field. More recently HL/LH have shown that about half the variance in the ECMWF height forecast errors at 6 hours over North America can be accounted for by components which are of very large scale in the horizontal, and have an almost barotropic structure in the vertical. The proportion of error accounted for by such deep structures probably varies between land and sea. This indicates that the choice of structure function should depend on data coverage. Such a possibility is more readily handled with the Kalman-Bucy formalism than with current operational systems.

An important point noted by $\mathrm{HL} / \mathrm{LH}$ is that it may be possible to get a more balanced analysis in mid-latitudes by analysing wind scaled by the sine of the latitude rather than the wind itself. This would give a more accurate treatment of the variation of the Coriolis parameter, and would probably be of most benefit in data sparse regions over the oceans.

\section{b) Satellite temperature retrievals}

Temperature soundings ("retrievals") from polar-orbiting satellites provide the only truly global three dimensional observing system. This data is of crucial importance to operational forecasting, especially over the oceans, Uppala et al. (1984), Kashiwagi (1984). Ongoing efforts are devoted to improving the quality and utilisation of the data, McMillin et al. (1979, 1982), Smith et al. (1985). In current operational practice the temperature and humidity retrievals are based on statistical regression of the radiances on collocated radiosondes. Each stage of the processing from earth-location, through calibration and cloud clearing, to the production of the final retrieval is the subject of extensive development work as discussed in the reports of the International TOVS study Conferences (Menzel and Lynch, 1985).

Current questions of most direct concern to Numerical Weather Prediction are related to the vertical and horizontal resolution of the information in the retrievals. We need to know how the spatial correlation of the errors in the retrievals depend on the type of first guess used for the retrieval, either shortterm climatology or a forecast model; and whether the retrieval should be one-dimen- 
sional or three-dimensional.

The operational resolution of the NESDIS retrievals is approximately $250 \mathrm{~km}$, but only a subset of this, with a resolution of $500 \mathrm{~km}$, is currently available on the GTS. Direct read-out of the radiances can provide average resolution of $80 \mathrm{~km}$ when the satellite is above the horizon. Examination of the radiance data (Kelly, 1985) suggests that there is considerable information on quite small scales in the horizontal. However the vertical resolution of the data is rather low. In clear sky conditions about 4 independent pieces of information are available in the vertical in the troposphere, while in cloudy conditions this reduces to about 2 . Historically, data users have demanded that the retrievals should look as much like radiosondes as possible, and the data producers have responded by producing retrievals which appear to offer more vertical resolution than is justifiable. This trend is being reversed as data users become more aware of the real capabilities of the instrumentation. If thicknesses for deep layers are the only reliable data in certain areas, then that is all one should use, (Pailleux and Kelly, 1987, pers. comm).

Two critical problems in generating a detailed vertical retrieval are the estimate of the surface temperature, and the estimate of the pressure of the tropopause. Low level clouds or inversions complicate the estimation of the surface temperature, particularly in the presence of snow or ice. Independent estimates of land surface and sea surface temperature can be very useful in anchoring the temperature of the lower layers, Phillips (1981a). Hammon and Bell (1985, pers. comm.) have demonstrated how the use of a statistical retrieval method can give rise to a very poor estimate of the upper troposphere and lower stratosphere temperature structure, when the true tropopause is at a quite different level from the mean tropopause of the set of sondes from which the retrieval coefficients were derived. The mean radiosonde provides the first-guess for the retrieval. Lorenc and Eyre (1985) have argued that if both the retrieval and the first guess are known then one can improve the retrieval if a better first guess becomes available (say from a forecast model). NESDIS has found that improvements near the tropopause are possible by stratifying the regression coefficients according to the brightness temperature at about $90 \mathrm{mb}$ in the 4 th microwave channel (Phillips, pers comm, 1987).

Much work is underway to establish a more direct connection between the raw radiance data and the assimilating model. Susskind (1985) and others have used the assimilating model to provide the first guess for the radiance field in a physical retrieval method. There is a danger in this procedure that the whole assimilation may drift away from the truth in data sparse areas. Kelly and Pailleux (1986, pers. comm.) have seen some evidence for this. Chedin (1984) avoids much of this danger by only using the model's forecast of surface pressure and, optionally, surface temperature as in the NESDIS retrievals (Phillips 1981a). The first-guess for the temperature in Chedin's approach is generated by an elaborate statistical procedure which picks a good first guess from a large library of observed atmospheric states. Durand (1985) has gone further than others in using the observed radiances directly in the $\mathrm{O} / \mathrm{I}$ calculation. The first guess for the radiances is generated by a radiative calculation from the first guess temperatures. This approach amounts to a three-dimensional retrieval algorithm.

\subsection{Analysis in the tropics}

The advent of operationally useful Numerical Weather Prediction in the Tropics has been slow. There have been scientific and technical reasons for the slow rate of development. The availability of the FGGE data has provided a great atimulus to this area, resulting in many studies of the phenomenology of the tropical weather, and a deep study of the problems of objective analysis, initialisation and forecasting in the Tropics (National Research Council, 1985). These studies have had a beneficial effect on operational practice. Taken with improvements in modelling, we expect continued improvement in the skill of Numerical Weather Prediction in the tropics. 


\section{a) Analysis and data problems}

Reed et al. (1986) have examined the performance of the ECMWF system in AugustSeptember 1985 in the West African-Atlantic sector. They found that the analyses showed a reasonable level of skill when data was available. They also found many aspects of the observing system and analysis system which could be improved. The forecasts to two days showed good skill in the initiation, evolution and propagation of African Waves. Subjective evaluations of forecasts in the West Pacific between June and October 1985 (Pasch pers. comm. 1985, WMO 1986b) also indicate that, in some instances, there is useful forecast skill to 2 or 3 days at low levels and perhaps as much as 5 days at upper levels.

Gray et al. (1986) report that the trackforecast is the most important element in operational forecasting for tropical storms. Reed et al. (1986) report that forecasts for the vorticity maximum in tropical depressions and storms were frequently successful at two days when observational data was available. They also reported many cases when satellite imagery permitted an accurate fix of storm position, even though cloud track winds were unavailable or gave little indication of the presence of a storm. Kanamitsu (1985) reports that JMA use satellite imagery to generate synthetic ('bogus') data to represent tropical storms in a consistent way in their initial data. Extension of this approach to other areas would be worth investigation.

A companion paper in this volume (Puri and Gauntlett, 1986) reviews recent progress on analysis of the mass and wind fields in the tropics. Lack of data is the main difficulty in tropical analysis, but that is largely outside the control of operational centres. Of the problems that are within our ambit, the formulation of a suitable mass-wind relationship for analysis in the tropics is still unresolved (Daley, Cats and Wergen, 1986). Phillips (1986) has calculated the statistics of an ensemble of slow modes in mid-latitudes, and shown that they provide a convenient vehicle to organise the statistics necessary for $\mathrm{O} / \mathrm{I}$. An equivalent calculation on an equatorial beta-plane yields an ensemble whose statistics are inherently non-homogeneous, and therefore difficult to use. Approaches using least squares Hough-function fitting also have difficulties (Halberstam, 1985; Daley, 1983). Aliasing between Rossby and Kelvin modes is therefore a continuing problem (Cats and Wergen, 1982); Daley, Cats and Wergen, 1986). The problems of analysing the large scale wind field, also discussed by Daley, Cats and Wergen (1986), are much reduced if one incorporates large scale terms in the correlation model to allow for the occurrence of forecast errors on very large scales (HL/LH).

Reed et al. (1986) and Julian (1980) both discuss cases where the analysis of the divergent wind field in the tropics was defective. Two approaches to the problem have been used in one-level calculations. Julian (1985) uses outgoing long wave radiation as a proxy for the velocity potential field. Daley (1985) uses the method of $\mathrm{O} / \mathrm{I}$ with a relaxed nondivergence constraint. Both methods show promising results, but have still to be tested in multi-level assimilation.

\section{b) Humidity and the initialisation of moist processes}

The definition of the humidity field is one of the most important and difficult problems in tropical analysis. The results of the analysis can be critical for the success of forecasts or the success of diagnostic calculations. The data coverage is as bad for humidity as for winds. Chen (1984) studied the humidity fields in the ECMWF and GFDL analyses of the main FGGE IIb dataset. He found substantial differences in the zonally averaged humidity budgets derived from the ECMWF and GFDL analyses. The differences were consistent with the known differences in the intensity of the Hadley circulation in the two sets of analyses, arising from the use of adiabatic normal mode initialisation at ECMWF but not at GFDL. More recently McGuirk et al. (1986) have studied the phenomenon of 'moisture burst' in the eastern Pacific, and has found good qualitative agreement between the FGGE analyses and independent satellite imagery not used in the analyses. 
Apart from the radiosondes, the most widely distributed data sources are the humidity retrievals from the TOVS instruments; at best this can give two or three useful numbers in the vertical. As discussed by Cadet (1983), this data is not available in cloudy areas; he found it useful to introduce some bogussing of data to upgrade the FGGE analyses in areas of persistent cloudiness over the Indian Ocean.

The quality of the TOVS humidity data has been the subject of a number of recent investigations (Johnson, 1984 ; Illari 1986). Illari concluded on the basis of a variety of collocation studies, that the TOVS data were not as good as radiosonde data, but were at least comparable in quality. The TOVS humidity data is used in the French operational system since 1984, and also at ECMWF since early 1986. Some efforts to use other types of satellite data as proxy data for humidity, including cloud top temperatures and histograms of cloud-top temperature, have been reported. Kanamitsu (1984) reports a beneficial impact of the use of the latter data.

The balance between the dynamics and the diabatic forcing is critical to forecast success in the Tropics. The most elaborate and determined efforts to achieve this balance have been reported by Krishnamurti et al. (1984). They used rainfall rate data and cloud-top temperature data to compile an estimate of the field of precipitation at the initial time of a forecast. They then made adjustments to the initial data in their model to ensure i) a balance between adiabatic warming and radiative cooling in the extensive subsidence areas of the tropics and ii) an initial rainfall rate in accordance with the observations in the rain areas. They found a substantial benefit from this initialisation of the diabatic forcing, a benefit which could be found in the forecasts well into the medium range. Illari (1986) also indicates that improvements in the humidity analysis have a distinct beneficial effect on tropical rainfall forecasts.

\subsection{Data quality control}

Improvements in Numerical Weather Pre- diction can come from improvements in technique across a wide spectrum of problems in analysis and modelling (Bengtsson 1985). A particularly critical problem is quality control of the observations presented to the analysis systems.

A striking example of the importance of quality control is discussed in the three-way analysis comparison in Hollingsworth et al. (1985). In that study the same set of FGGE II-b observational data was presented to advanced analysis systems at ECMWF, NMC Washington, and UKMO. This produced three sets of analyses which were used to initialise forecasts with the models in all three centres, resulting in 9 sets of forecasts. In one case the forecasts with all models from one of the analyses produced by day 3 a spurious low in the North east Pacific which was not found in the verification, nor in any of the forecasts from the other analyses. The critical diference was traced to an error in the quality control of aircraft data in that analysis. Quality control is clearly of vital importance for forecast success.

\section{a) The variability of operational data quality}

Data can be corrupt for a wide variety of reasons. We have seen aircraft and ships reporting position at $30^{\circ} \mathrm{E}$ over Africa when the true position was at $30^{\circ} \mathrm{W}$. Similar bugs can occur in earth-location of satellite reports. We have received 1-day old reports from a whole continent labelled as today's reports, because of software problems. Radiosondes can have serious biases in height or wind or both. Drifting buoys in isolated areas of the ocean can report constant pressures if the instrumentation or the telemetry fails. An operational centre has to develop a wide range of quality control tools to monitor the quality of the data, and to identify systematic problems as rapidly as possible. Random errors are always encountered, and real time quality control algorithms must be capable of dealing with them. Improvements in telecommunications are effective in reducing the number of errors due to corruptions in transmissions.

In operational practice, there are many different approaches to the quality control 
problem, as evidenced by the report of a recent workshop (ECMWF, 1984). In some Centres there is a heavy reliance on manual intervention to detect and correct errors, while others rely entirely on automated methods.

\section{b) Automated hierarchical quality control algorithms}

Most operational quality control systems have a hierarchical sieve structure, which seek to identify and exclude data with successively smaller gross errors, until the data finally used in the analysis calculation has errors which are within the normal range of random error. The ECMWF system (Lorenc, 1981) is typical in having such a hierarchical structure.

After a series of checks against climatology, the first-guess, and closely adjacent observations, the main check in the ECMWF system is against the combination of the other data and the first guess. The check begins with a preliminary analysis for the value of each datum, using all other data in the analysis volume except the datum being checked. If the datum departs too far from this estimate it is flagged. The worst flagged datum is then eliminated and all the doubtful data re-checked. The process is repeated until all the data are judged to be mutually consistent. By a clever choice of optimisation criterion Lorenc (1981) was able to calculate all the preliminiary analyses by making small corrections to the inverse of a single large matrix. The cost of the algorithm is therefore quite modest.

The cross-validation approach has proved to be a very effective device for deciding on rejection or acceptance of data. Wahba and Wendelberger (1980) used a similar algorithm, which they called generalised cross validation (GCV) for a related purpose. They examined the use of thin-plate splines, which are generalisations of smoothing splines, for analysis purposes. They used GCV to determine the setting of the observational noise and the setting of the 'roll-of' (curvature at the origin) of the analogue of the auto-correlation function, in order to get the best fit to the data which minimised a penalty function on cur- vature. In a sense both their algorithm and that of Lorenc use the same method to proceed in oposite directions-one uses the structure functions to quality control the data, while the other adjusts the structure function to fit the data.

The tuning of quality control algorithms requires reliable statistics on the magnitude of first-guess errors, of analysis errors, and of observation errors for all observation types. This information is easily generated from archives of data assimilation results.

\section{c) A Bayesian approach to quality control}

An inherent difficulty of a strictly hierarchical approach to quality control is that a datum which is rejected at an early stage in the processing is not re-considered at a later stage, when more extensive calculations might suggest that the datum is in fact free of gross error. There are many cases when it would be desirable to poll a set of tests of equal importance rather than to use the hierarchical approach (Gandin pers. comm. 1985).

Lorenc (1986), following Purser (1984), has proposed an approach to this problem based on Bayesian arguments, and using the principle of maximum likelihood. He begins by considering the probability distribution of the observation errors of a set of data where the errors may consist of two types. These are first the usual observation errors with Gaussian statistics and small variance. There is also a finite probability of gross error; the probability of such an error is constant across the range of climatologically possible states.

Lorenc developed a maximum likelihood analysis algorithm which is exactly equivalent to $\mathrm{O} / \mathrm{I}$ when the statistics are Gaussian. This formulation can however deal with non-Gaussian statistics. When applied to the quality control problem, the Bayesian approach provides a rational method for polling the results of many independent tests. In particular it is possible to use the approach to pool, or ballot, the results of several individually inconclusive tests in order to form a soundly based decision on quality, as suggested by Gandin. 


\section{Concluding remarks}

This review has considered current and projected work on objective analysis under three main headings: theory, verification and practice. It is necessary to mention also a basic requisite for practical work. As assimilation systems have become more complex, it has been necessary to develop extensive archives and diagnostic facilities to monitor the behaviour of the systems. At ECMWF we keep a complete record of the processing of every single datum. This archive has been an essential raw material, and a vital simulus, for the development of new diagnostics of assimilation system performance.

Recent developments in the theory suggest that there are close links between all analysis methods based on optimality principles. Moreover, simpler analysis methods such as successive correction can be modified to converge to an optimal method in simple circumstances. Applications of the theory to four-dimensional analysis using the adjoint approach is likely to be an area of great activity. The basic job of any analysis algorithm is to filter the observation error from the data and interpolate the filtered data to a regular grid. We have presented a new viewpoint on $\mathrm{O} / \mathrm{I}$ which shows the algorithm in a simple light with an intimate connection between the filtering and interpolation capabilities. The abundance of new ideas, coupled with the general awareness of the importance of analysis accuracy for NWP, augurs well for productive work in the next few years.

Verifications of observations, forecasts, and analyses have been reviewed, using statistics on one-point and two-point correlations of climatic fluctuations, of forecast errors, and of the differences between observations and analyses. The two-point correlations are inter-related through the kinematic equations of two-dimensional turbulence; in the limit of vanishing spatial lag they degenerete to the one-point correlations.

The kinematic diagnostics offer a means to compare the error characteristics of different observing systems. They already indicate some sampling problems with cloud- track wind data, and further work remains to be done. When used to verify short-range forecasts, the diagnostics provide a complete description of the structure of the forecast errors. A simple theory of forecast errors can reproduce many of the features of the empirically determined structures. New results indicate that there may be a need for anisotropic and non-separable correlation functions for $\mathrm{O} / \mathrm{I}$ analysis.

The correlation diagnostics offer new and rigorous ways to estimate analysis error at observation points. They provide a quick and easy measure of the efficiency of an analysis system. They should prove useful in identifying the reasons for system dependence in the results of observing system experiments. In time they ought to enable one to prove that a three-dimensional algorithm has used all the data to the fullest extent of its capability. At that point we shall need the more explicity four dimensional adjoint method if progress is to continue.

The review of current practice considered first the special problems of analysis in data rich areas over land and near mountains. The problems of analysis over the mid-latitude oceans were considered next, and then the problems of tropical analysis. Extensions of $\mathrm{O} / \mathrm{I}$ offer new approaches to these problems which will be explored in the near feture. Some problems will not yield to this approach because of their inherent unhomogeneity. In the longer term, new developments in the theory of 4-dimensional analysis may well supersede current methods. Finally we considered developments in data quality control, an area of great importance for all other developments. New developments here, using Bayesian methods, offer promise.

The field of objective analysis for Numerical Weather Prediction is enjoying rapid development as a result of progress in observing and computing technology, and of theoretical advances in analysis method. The area is interesting and challenging, as it is the meeting point of many different areas of study: observational technology, statistical approximation theory, initialisation theory, numerical methods, dynamics, and the parameterisation 
of physical processes. The importance of accuracy in the analyses for forecasting and for diagnostic studies adds zest to the work.

The application of quantitative method to all aspects of assimilation has produced encouraging results. We may confidently expect progress to continue. The growing recognition that objective analysis is as exciting a challenge as any of the more glamorous areas of atmospheric science will help attract new workers for the problems to be faced over the next decade.

\section{Acknowledgements}

I am grateful to my mentors A.C. Lorenc, N.A. Phillips, P. Lönnberg and R. Daley for an education in analysis. I am greatful to present and former colleagues, and to visitors to the ECMWF Data Assimilation and Meteorological Operations Groups, for valuable discussions. Thanks are also due to the many participants in analysis workshops and seminars at ECMWF, where problems have been thrashed through to good effect. The manuscript was much improved by comments from D. Anderson, G. Kelly, P. Lönnberg, N.A. Phillips, A.J. Simmons and P. Unden. Mrs. M. Simpson and Mr. R, Hine did excellent work on the manuscript and the illustrations. Finally I am grateful to P. Lönnberg, for use of unpublished results.

\section{References}

Andersen, J.H., 1982: Experiments with increased vertical resolution in the ECMWF analysis system. In Proceedings of ECMWF Workshop on 'Current Problems in Data Assimilation', pp. 183206 ; available from ECMWF.

Arpe, K., 1980: Confidence limits for verification and energetics studies. ECMWF Tech Rept. 18; available from ECMWF.

- 1983: Diagnostic evaluation of analysis and forecasts : climate of the model. In Proceedings of ECMWF Seminar on 'Interpretation of Numerical Weather Prediction Products', pp. 99140 ; available from ECMWF.

$\longrightarrow$, A. Hollingsworth, A.C. Lorenc, M.S. Tracton, S. Uppala and P. Kallberg, 1985: The response of Numerical Weather prediction Systems to FGGE II-b Data, Part II: Forecast Verifications and Implications for Predictability. Quart. J. Roy. Meteor. Soc., 111, 67-102.
Atlas, R., E. Kalnay, W. E. Baker, J. Susskind, D. Reuter and M. Halem, 1984: Observing system experiments at GLAS. In Proceedings of ECMWF /WMO Seminar on 'Data Assimilation Systems and Observing System Experiments with Particular Emphasis on FGGE', Vol. I pp. 353-376; available from ECMWF.

Backus, G.E. and J.F. Gilbert, 1968: The resolving power of gross earth data. Geophys. J. Roy. Astron. Soc., 16, 169-205.

Baede, A.P.M., S. Uppala and P. Kallberg, 1987 : Impact of Aircraft Wind Data on ECMWF Analyses and Forecasts during the FGGE period 8-19 Nov. to appear in Quart. J. Roy. Met. Soc., July 1987.

Baer, F., 1977: Adjustment of initial conditions required to suppress gravity oscillations in nonlinear flows. Beitr. Phys. Atmos., 50, 35.-366.

Baker, W.E., 1983: Objective analysis and assimilation of observational data from FGGE. Mon. Wea. Rev., 111, 328-342.

- R. Atlas, M. Halem and J. Susskind, 1984 : A case study of forecast sensitivity to data and data analysis technique. Mon. Wea. Rev., 112, 1544-1561.

Baker, W., S. C. Bloom, J. S. Woolen, M. S. Nestler, E. Brin, T.W. Schlatter and G.W. Branstator, 1987: Experiments with a three-dimensional statistical objective analysis scheme using FGGE data. Mon. Wea. Rev. 115, 273-296.

Balgovind, R., A. Dalcher, M. Ghil and E. Kalnay, 1983: A stochastic-dynamic model for the spatial structure of forecast error statistics. Mon. Wea. Rev., 111, 701-722.

Barwell B. R. and A.C. Lorenc, 1985: A study of the impact of aircraft wind observations on a large scale analysis and numerical weather prediction system. Quart. J. Roy. Meteor. Soc., 111, 103-129.

Barnes, S.L., 1964: Mesoscale objective map analysis using weighted time-series observations. NOAA Tech. Memo. ERL NSSL-62. (NTIS COM73-10781)

Batchelor, G.K., 1953: The Theory of Homogeneous Turbulence. Cambridge University Press.

Bengtsson, L., 1975: Four-dimensional assimilation of meteorological observations. GARP Publ. Ser. 15.

1978: Growth rate and vertical propagation of the initial error in baroclinic models. Tellus, 30, 323-334.

and A. J. Simmons, 1983: Medium range weather prediction-operational experience at ECMWF; in 'Large Scale Dynamical processes in the Atmosphere' ed. B. J. Hoskins and R.P. Pearce, Academic Press xvi+397 pp. 
1985: Medium-range forecasting at the ECMWF. Advances in Geophysics, 28, Academic Press, 3-51.

Bergman, K., 1978: Role of observational errors in optimum interpolation analysis. Bull. Am. Meteor. Soc., 59, 1603-1611

Bergman, K.H., 1979: Multivariate analysis of temperatures and winds using optimum interpolation. Mon. Wea. Rev., 107, 1423-1444.

- and W.D. Bonner, 1976: Analysis error as a function of observation density for satellite temperature soundings with spatially correlated errors. Mon. Wea. Rev., 104, 1308-1316.

Bergthorsson, P. and B.R. Döös, 1954: Numerical weather map analysis. Tellus, 7, 329-340.

Bourke, W., R. Seaman and K Puri, 1985: Data Assimilation, in Advances in Geophysics Vol. 28B : Weather Dynamics, ed. S. Manabe. pp. 124149. Academic Press.

Bratseth, A. M., 1986: Statistical interpolation by means of successive corrections. Tellus, 38A, 439-447.

Bromley, R., 1985: Observing system experiments using the Meteorological Office's 15-level model. In Proceedings of ECMWF/WMO Seminar on 'Data Assimilation Systems and Observing System Experiments with Particular Emphasis on FGGE', Vol. I pp. 277-326; available from ECMWF.

Brown, P.S. and G. D. Robinson, 1979: The variance spectrum of tropospheric winds over Eastern Europe. J. Atmos. Sci., 36, 270-286.

Buell, C.E., 1971: Two-point wind correlations on an isobaric surface in a nonhomogeneous nonisotropic atmosphere. J. Appl. Met., 10, 12771274 .

- 1972a: Correlation functions for wind and geopotential on isobaric surfaces. $J$. Appl. Met., 11, 51-59.

- , 1972b: Variability of wind with distance and time on an isobaric surface. J. Appl. Met., 11. 1085-1091.

- and R.S. Seaman, 1983: The 'scissors' effect : anisotropic and ageostrophic influences on wind correlation coefficients. Aust. Met. Mag., 31, 77-83.

Burridge, D. M. and J. Haseler, 1977: A model for medium range weather prediction-adiabatic formulation. ECMWF Tech Rept 4, available from ECMWF.

Buzzi, A., A. Trevisan and E. Tosi, 1984: A preliminary isentropic analysis of lee cyclogenesis during ALPEX. Riv. Meteor. Aero. XLIV, 69-78.

Cadet, D., 1983: Mean fields of precipitable water over the Indian Ocean during the 1979 summer monsoon from TIROS-N soundings and FGGE data. Tellus, 35A, 329-345.

Cats, G. J. and W. Wergen, 1982: Analysis of large scale normal modes by the ECMWF analysis scheme. In Proceedings of ECMWF Workshop on 'Current Problems in Data Assimilation', pp. 343372 ; available from ECMWF.

Chen, T.-C., 1985: Global water vapour flux and maintenance during FGGE. Mon, Wea. Rev., 113, 1801-1819.

Cressman, G.P., 1959: An operational objective analysis system. Mon. Wea. Rev., 87, 367-374.

Chedin, A., N.A. Scott, C. Wahiche and P. Moulinier, 1985: The improved initialisation inversion method: a high resolution physical method for temperature retrievals from the TIROS-N series. J. Clim. Appl. Meteor., 24, 128-143.

Courtier, P., 1985: Experiments in data assimilation using the adjoint model technique. In Proceedings of ECMWF Workshop on High Resolution Analysis', pp. 299-233; available from ECMWF.

Daley, R., 1978: Variational non-linear normal mode initialisation. Tellus, 30, 201-208.

- 1981: Normal Mode Initialisation. Rev. Geophys. Space. Phys., 19, 450-468.

- 1983: Spectral characteristics of the ECMWF objective analysis system. ECMWF Tech. Rep. No. 40. 119 pp.

, 1985: The analysis of synoptic scale divergence by a statistical interpolation procedure. Mon. Wea. Rev., 113, 1066-1079.

-, G.J. Cats and W. Wergen, 1986: The objective analysis of planetary scale flow. Mon. Wea. Rev., 114, 1892-1908.

Dell'Osso, L., 1984: High resolution experiments with the ECMWF model. Mon. Wea. Rev., 112, 1853-1883.

Delsol, F., 1985: Monitoring the availability and quality of observations at ECMWF. Proc of ECMWF Workshop on the Use and Quality Control of Meteorological Observations 55-89. Available from ECMWF Reading U.K.

Dey, C. and L.L. Morone, 1985: The evolution of the NMC Global Assimilation System : Jan. 1982Dec. 1983. Mon. Wea. Rev., 113, 304-318.

DiMego, G., P.A. Phoebus and J.E. McDonnell, 1984: Data processing, quality control and selection for optimum interpolation analyses at the National Meteorological Centre. In Proceedings if ECMWF Workshop on 'The Use and Quality Control of Meteorological Observations for $\mathrm{Nu}$ merical Weather Prediction', pp. 325-368; available from ECMWF.

- 1985: The NMC regional analysis system. In Proceedings of ECMWF workshop on 'High 
Resolution Analysis', pp. 213-258; available from ECMWF.

Drozdov, O.A. and A.A. Shepelevskii, 1946: The theory of interpolation in a stochastic field of meteorological elements and its application to meteorological map and network rationalisation problems. Trudy NIU GUGMS Series 1, No. 13.

Durand, Y., 1985: The use of satellite data in the French high resolution analysis. In Proceedings of ECMWF Workshop on 'High Resolution Anal$y$ sis', pp. 89-128; available from ECMWF.

ECMWF, 1984: Seminar/Workshop on Data Assimilation and Observing System Experiments with Particular Emphasis on FGGE, 2 Vols.; available from ECMWF, Shinfield Park, Reading U. K.

Eliassen, A., 1954: Provisional report on calculation of spatial covariance and autocorrelation of the pressure field. Rap. No. 5 VidenskapsAkademiets Institutt for Vaer og Klimaforskning, Oslo, Norway.

Flattery, T., 1970: Spectral models for global analysis and forecasting. Air Weather Service Tech. Rep. 242, pp. 42-54. U.S. Naval Academy. Amnnapolis, Md.

Franke, R., 1985: Sources of error in objective analysis. Mon. Wea. Rev., 113, 260-270.

- and Gordon, W.J., 1983: The structure of optimum interpolation functions. Naval Postgraduate School, Monterey, Calif. NPS-53-83-0005 (Feb. 83),

Gandin, L.S., 1963: Objective analysis of meteorological fields. Translated from Russian by the Israeli Program for Scientific Translations (1965).

Ghil, M., S. Cohn, J. Tavantzis, K. Bube and E. Isaacson, 1981: Applications of estimation theory to numerical weather prediction. in Dynamical Meteorology Data Assimilation Methods, ed. L. Bengtsson, M. Ghil, E. Källén, pub Springer, pp. 139-224.

Gilchrist, A. (ed.), 1982: JSC Study Conference on Observing Systems Experiments, Exeter, April 19-22 1982. Report 4 of the Numerical Experimentation Programme, pub GARP and WCRP, WMO, Geneva.

Gray, W.M., 1986: Summary of the WMO sponsored international workshop on tropical cyclones. Bull. Am. Met. Soc., 67, 849-850.

Girard, C. and M. Jarraud, 1982: Short and Medium Range Forecast differences between a spectral and grid-point model. An extensive quasi-operational comparison: ECMWF Tech. Rept. 32, available from ECMWF.

Gustafsson, N., 1981: 'A review of methods for objective analysis' in Dynamic meteorology-Data assimilation methods ed. L. Bengtsson, M. Ghil,
E. Kallen, Springer-Verlag,

Guymer, L. B., 1978: Operational application of satellite imagery to synoptic analysis in the southern hemisphere. Tech. Rept. 29. Bureau of Meteorology, Australia.

Halberstam, I. and S.L. Tung, 1984: Objective analysis using Hough vectors evaluated at irregularly spaced locations. Mon. Wea Rev., 112, 1804-1817

Heckley, W.A., 1985: Systematic errors of the ECMWF operational forecasting model in the tropics. Quart. J.R. Met. Soc., 111, 709-738.

Hollett, S. R., 1975: Three dimensional spatial correlations of $\mathrm{PE}$ forecast errors. M.Sc. thesis. Department of Meteorology, McGill University, Montreal, Canada. $73 \mathrm{pp}$.

Hollingsworth, A., K. Arpe, M. Tiedtke, M. Capaldo and H. Savijarvi, 1980: The performance of a medium-range forecast model, in winter-impact of physical parameterizations. Mon. Wea. Rev., 108, 1736-1773.

, 1984: Lecture Notes on Analysis (Ch. 6), available from ECMWF, Shinfield Park Reading, U. K,

- A. C. Lorenc, M.S. Tracton, K. Arpe, G. Cats, S. Uppala and P. Kallberg, 1985: The response of Numerical Weather prediction Systems to FGGE II-b Data Part I: Analyses. Quart. J. Roy. Met. Soc., 111, 1-66.

, D. B. Shaw, P. Lönnberg, L. Illari, K. Arpe and A. J. Simmons, 1986: Monitoring of observation quality by a data assimilation system. Mon. Wea. Rev., 114, 861-879.

and P. Lönnberg, 1986: The statistical structure of short range forecast errors as determined from radiosonde data. Part I The wind errors. Tellus, 38A, 111-136.

and 1987 : The verification of objective analyses. ECMWF Tech. Memo.

Holopainen, E. O., 1978: On the dynamic forcing of the long-term mean flow by the large scale Reynold's stresses in the atmosphere. J. Atmos. Sci., 35, 1596-1604.

Hoskins, B. J., I. N. James and G. H. White, 1983: The shape, propagation and mean-flow interaction of large scale weather systems. J. Atmos. Sci., 40, 1595-1612.

and P.D. Sardeshmukh, 1987: Transient eddies and the seasonal mean rotational flow. J. Atmos. Sci., 44, 328-338.

Hutchings, J. W., 1955: Turbulence theory applied to large-scale atmospheric phenomena. J. Met., 12, 263-271.

Ikawa, M., 1984a : An alternative method of solving the weak constraint and a unified expression of weak and strong constraints in variational 
objective analysis. Papers in Met. and Geopeys., 35, 71-79.

- 1985b: Generalisation of multivariate optimum interpolation method and the roles of linear constraint and covariance matrix in the method. Part I : in discrete form. Papers in Met. and Geophys., 35, 81-102.

- 1985 c : Genealisation of multivariate optimum interpolation method and the roles of linear constraint and covariance matrix in the method. Part II : in continuous form. Papers in Met. and Geophys., 35, 169-180.

Illari, L., 1986: The quality of ECMWF humidity analysis. Proc ECMWF workshop on High Resolution Analysis, pp. 41-68; available from ECMWF.

Jarraud, M., A. J. Simmons and M. Kanamitsu, 1985: Development of the high resolution model. ECMWF Tech. Memo. 107. Available from ECMWF, Shinfield Park, Reading, Berkshire, RG2 9AX, England.

Jaszwinski, A.H., 1970: Stochastic Processes and Filtering Theory. Academic Press, xiv +376 .

Johnson, D.S., 1984: Meteorological parameters derived from space-based observing systemsFGGE and after. Procs ECMWF Seminar on Data Assimilation and Observing System Experiments with Particular Emphasis on FGGE, Vol. I pp. 47-108; available from ECMWF, Shinfield Park, Reading U.K.

Julian, P. R. and H. J. Thiebaux, 1975: On some properties of correlation functions used in optimum interpolation schemes. Mon. Wea. Rev., 103, 605-616.

- 1980: An evaluation of the FGGE tropical observing system. Proc. Int. Conference on FGGE data analysis and results. Bergen, pp. 239-246. available from WMO, Geneva.

- 1983: On the use of observation error data in FGGE Main Level III-b analysis. ECMWF Research Department Tech. Memo. No. 76. 24 pp.

Julian, P., 1984: Objective analysis in the tropics: a proposed scheme. Mon. Wea. Rev., 112, 17521767.

Kallberg, P., S. Uppala, N. Gustafsson and J. Pailleux, 1982: The impact of cloud track wind data on global analyses and medium range forecasts. Tech. Rep. $34 E C M W F$, available from ECMWF.

- and F. Delsol, 1986: Systematic biases in cloud-track-wind data from jet stream regions. Programme on Short and Medium Range Numerical Weather Prediction Research Tokyo pp. 15-18. WMO PSMP Rept 19, available from WMO, Geneva.

Kanamitsu, M., 1984: Data processing, selection and quality control, as used at JMA. Proc
ECMWF Workshop on The Use and Quality Control of Meteorological Observations, pp. 368-296; available from ECMWF, Shinfield Park, Reading U. K.

von Karman, T. and L. Howarth, 1938: On the statistical theory of isotropic turbulence. Proc. Roy. Soc. A, 164, 192-219.

Kelly, G. A., 1985: The use of satellite radiance measurements in the ECMWF analysis system. In Proceedings of ECMWF Workshop on High Resolution Analysis, pp. 27-40; available from ECMWF.

Khinchin, A. N., 1934: Korrelationstheorie der stationaren stochastischen Prozesse, Math. Ann. 109, No. 4, 604-615.

Kimeldorf, G. and G. Wahba, 1970: A correspondence between Bayesian estimation on stochastic processes and smoothing by splines. Ann. Inst. Statist. Math., 41, 495-502.

Kistler, R.E. and D.F. Parrish, 1982: Evolution of the NMC data assimilation system : September 1978-January 1982. Mon. Wea. Rev., 110, 13351346.

Kashiwagi, K., 1984: On the impact of spacebased observing systems in the JMA global forecast/analysis system. In Proceedings of ECMWF/WMO Seminar on 'Data Assimilation Systems and Observing System Experiments with Particular Emphasis on FGGE', pp. 229276; available from ECMWF.

Kitade, T., 1983: Nonlinear normal mode initialisation with physics. Mon. Wea. Rev., 111, 2194-2213.

Krishnamurti, T. N., R. Pasch, H. Pau, S. Chu and K. Ingles, 1983: Details of low-latitude numerical weather prediction using a global spectral model I. J. Met. Soc. Japan, 61, 188-207.

, K. Ingles, S. Cocke, R. Pasch and T. Kitade, 1984: Details of low-latitude numerical weather prediction using a global spectral model II. J. Met. Soc. Japan, 62, 188-207.

, 1985: Numerical weather prediction in low latitudes. In 'Advances in Geophysics Vol 28B-Weather Dynamics' ed S. Manabe, pp. 283331, Academic Press.

Larsen, G. et al., 1977: Analysis error calculations for the FGGE. ECMWF Internal Report No. 11.

Lau, N.-C., 1984: A comparison of circulation statistics based on FGGE level III-b analyses produced by GFDL and ECMWF for the special observing periods, NOAA data report ERL $G F D L-6$; available from NOAA, Executive Blvd, Rockville Md.

Leith, C.E., 1973: The standard error of timeaverage estimates of climatic means. J. Appl. Met., 12, 1066-1069. 
1980: Non-linear normal mode initialisation and quais-geostrophic theory. J. Atmos. Sci., 37, 958-968.

, 1984 : Statistical methods for verification of long and short range weather forecasts. pp. 207-229 of Problems and prospects in long and medium range weather forecasting; ed D.M. Burridge and E. Källén, Springer.

Le Dimet, F.X. and O. Talagrand, 1986: Variational algorithms for analysis and assimilation of meteorological observations: theoretical aspects. Tellus, 38a, 97-110.

Lewis, J. M. and J.C. Derber, 1985: The use of adjoint equations to solve a variational adjustment problem with advective constraints. Tellus, 37A, 309-322.

Lönnberg, P., 1982: Structure functions and their implications for higher order analysis. pp. 143178 ECMWF Workshop on Current Problems in Data Assimilation, available from ECMWF.

and D.B. Shaw, 1984: Data selection and quality control in the ECMWF analysis system. Proc ECMWF Workshop on The Use and Quality Control of Meteorological Observations, pp. 225254; available from ECMWF, Shinfield Park, Reading U.K.

and A. Hollingsworth, 1986: The statistical structure of short range forecast errors as determined from radiosonde data. Part II Covariance of height and wind errors. Tellus, 38A, 137-161.

Lorenc, A.C., 1981: A global three-dimensional multivariate statistical interpolation scheme. Mon. Wea. Rev., 109, 701-721.

, 1984: Analysis methods for the quality control of observations. In Proceedings of ECMWF Workshop on 'The Use and Quality Control of Meteorological Observations for Numerical Weather Prediction', pp. 397-428; available from ECMWF.

, 1986: Analysis methods for numerical weather prediction. Quart. J. Roy. Met. Soc., 112, pp. 1177-1194.

- W. Adams and J. Eyre, 1985: The analysis of high resolution satellite data in the Meteorological Office. In Proceedings of ECMWF Workshop on 'High Resolution Analysis', pp. 6988; available from ECMWF.

Lorenz, E. N., 1982: Atmospheric predictability experiments with a large numerical model. Tellus, 34, 505-513.

Lyne, W.H., R. Swinbank and N.T. Birch, 1982 : A data assimilation experiment and the global circulation during the FGGE special observing periods. Quart. J. Roy. Meteor. Soc., 108, 575609 .
Machenhauer, B., 1977 : On the dynamics of gravity oscillations in a shallow water model, with application to normal mode initialisation. Contrib. Atmos. Phys., 50, 253-271.

Marchuk, G.I., 1975: Formulation of the theory of perturbations for complicated models. Part II, Weather Prediction. Geofisica Internacional, 15, pp. 169-182.

Marr, D., 1979: Vision, Freeman and Sons, San Francisco, pp. 467.

McGinley, J., 1985a : Meteorological analysis using the calculus of variations (variational analysis). Riv. Meteor. Aero. XLIV, 37-44.

, 1985b: Analysis of flow blocking during ALPEX by imposition of mass continuity. Riv. Meteor. Aero. XLIV, 95-102.

- and J.S. Goerrs, 1986: Effects of terrain height and blocking initialisation on numerical simulation of Alpine lee cyclogenesis. Submitted for publication.

McGuirk, J. P., A. H. Thompson and L. L. Anderson, 1986: Wintertime disturbances in the tropical Pacific: FGGE IIIb and Satellite comparisons. in Proc of Nat. Conf. on Scientific results of the First Garp Global Experiment. Miami 1986, pub Amer. Meteor. Soc.

McMillin, L., D.G. Gray, H.F. Drahos, M.W. Chalfant and C.S. Novak, 1983: Improvements in the accuracy of operational satellite soundings. J. Clim. Appl. Meteor., 22, 1948-1955.

McPherson, R., K. H. Bergman, R. E. Kistler, G.E. Rasch and D.S. Gordon, 1979: The NMC operational global data assimilation system. Mon. Wea. Rev., 107, 1445-1461.

Menke, W., 1984: Geophysical Data Analysis: Discrete Inverse Theory. Academic Press pp. 260.

Menzel, W. P. and M. J. Lynch, 1985: A Report on the second international TOVS study conference. International Radiation Commission of IAMAP.

Miyakoda, K. and R.W. Moyer, 1968: A method of initialisation for dynamical weather forecasting. Tellus, 20, 115-128.

- 1985: Assessment of results from different analysis systems. In Proceedings of WMO/ ICSU Conference the Scientific Results of FGGE. pub WMO Geneva.

- J. Ploshay and W. Stern, 1986: Revised four-dimensional analysis scheme at GFDL and the preliminary study for re-analysis of FGGE data. In Proceedings of U.S. National Conference on the Scientific Results of the First GARP Global Experiment, pp. 21-23; American Meteorological Society, 45 Beacon Street, Boston, Mass. 1986 Miami meeting.

National Research Council, 1985: Proceedings of 
the First National Workshop on the Global Weather Experiment. National Academy Press.

Obukhov, A.M., 1954: Statistical description of continuous fields. Trudy Geofiz In-ta Akad Nauk SSSR No. 24(151) 3-42. English translation by Liaison Office, Technical Information Centre, Wright-Patterson AFB F-TS-9295/v.

Panchev, S., 1971: Random functions and turbulence. Pergamon Press pp. 444.

Pierrard, M.C., 1985: Intercomparison between cloud-track-winds and radiosonde winds. In Proceedings of Fifth Meteosat User conference, Rome, pub. European Space Agency.

Phillips, N.A., 1971: Ability of the Tadjbaksh method to assimilation temperature data in a meteorological system. J. Atmos. Sci., 28, 13251328.

, 1979: The nested grid model. NOAA Tech Report, NWS 22, 80 pp. Available from NOAA, Rockville, Md.

- 1981a: Cloudy winter satellite temperature retrievals over the extratropical northern hemisphere oceans. Mon. Wea. Rev., 109, 652659.

- 1981b: Variational analysis and the slow manifold. Mon. Wea. Rev., 109, 2415-2426.

-, 1982: On the completeness of multivariate optimum interpolation for large-scale meteorological analysis. Mon. Wea. Rev., 110, 1329-1334.

- 1986 : The spatial statistics of random geostrophic modes and first-guess errors. Tellus, 38A, 314-332.

Poggio, T., V. Torre and C. Koch, 1985: Computational vision and regularisation theory. Nature, 317, 314-319.

Puri, K., 1983: The relationship between convective adjustment, Hadley circulation and normal modes in the ANMRC spectral model. Mon. Wea. Rev., 111, 23-33.

- W. Bourke and R. Seaman, 1985: The impact of FGGE observing systems in the southern hemisphere. Proc First National Workshop on the Global Weather Experiment, Vol. II(i) pp. 146-160 National Academy Press.

- and D. J. Gauntlett, 1987: Numerical Weather Prediction in the Tropics, in this volume.

Purser, R. J., 1984: A new approach to the optimal assimilation of meteorological data by iterative Bayesian analysis. Preprints, 10th Conference on Weather Forecasting and Analysis. Pub. Am. Met. Soc., 102-105.

Reed, R. J., A. Hollingsworth, W. A. Heckley and F. Delsol, 1986: An evaluation of the performance of the ECMWF operational forecasting system in analysing and forecasting tropical Easterly Wave Disturbances. ECMWF Tech. Rept. 58; available from ECMWF.

Reimer, E., 1985: Analysis of ALPEX data. Proc ECMWF Workshop on High Resolution Analysis, pp. 155-182; available from ECMWF, Shinfield Park, Reading U.K.

Riehl, H., 1979: Climate and Weather in the Tropics. Academic Press pp. 611.

Rinne, J. and Frisk, K., 1979: The development of the analysis error of the $500 \mathrm{hPa}$ height, 19461969. Tellus, 31, 387-399.

- and S. Jarvenoja, 1983: Estimation of the analysis error variance of the $500 \mathrm{mb}$ height analyses in the northern hemisphere 1946-79. Mon. Wea. Rev., 111, 1404-1417.

- and $\longrightarrow, 1984$ : Random analysis error of the $500 \mathrm{mb}$ height analyses for the FGGE year. Mon. Wea. Rev., 112, 1389-1397.

and -1985 : Auto-correlation functions computed from daily $500 \mathrm{mb}$ geopotential height analyses. Mon. Wea. Rev., 113, 16811691.

Ritter, B., 1984: The impact of an alternative treatment of infrared radiation on the performance of the ECMWF forecast model. Proc IAMAP International Radiation Symposium 21-29 August, Italy pp. 277-280, Pub Deepak Publ.

Rutherford, I.D., 1972: Data assimilation by statistical interpolation of forecast error fields. J. Atmos. Sci., 29, 809-815.

Sasaki, Y., 1958: An objective analysis based on the variational method. J. Met. Soc. Japan, 36, 77-88.

, 1970 : Some basis formalisms in numerical variational analysis. Mon. Wea. Rev., 98, 875883.

Schlatter, T.W., 1975: Some experiments with a multivariate statistical objective analysis scheme. Mon. Wea. Rev., 103, 246-257.

, 1981: An assessment of operational. TIROS-N temperature retrievals over the United States. Mon. Wea. Rev., 109, 101-110.

Seaman, R.S., 1977: Absolute and differential accuracy of analyses achievable with specified observational network characteristics. Mon. Wea. Rev., 105, 1211-1222.

, 1983: Objective analysis accuracies of statistical interpolation and successive correction schemes. Aust. Meteor. Mag., 31, 225-240.

- and M.F. Hutchinson, 1985: Comparative real data tests of some objective analysis methods by witholding observations. Aust. Met. Mag., $33,37-46$.

Shaw, D. B., P. Lönnberg and A. Hollingsworth, 1984: The 1984 revision of the ECMWF as- 
similation system. Tech. Memo. 92, available from ECMWF.

- - and P. Unden, 1987 :

The 1984/85 revisions of the ECMWF assimilation system. Quart. J. Roy. Met. Soc. April 1987.

Simmons, A. J. and M. Jarraud, 1984: The design and performance of the new ECMWF operational model. Proc. ECMWF Seminar on 'Numerical Methods for Weather Prediction' Vol. 2, pp. 113-164, available from ECMWF.

Smith, W. L., H. M. Woolf, C. M. Hayden and A. J. Schreiner, 1985: The simultaneous retrieval export package. Proc. Second Int. TOVS Study Conf. Igls, Feb., 18-22.

Spalding, T.R. and Shaw, D. B., 1977 : Spatial correlations for Gate observations. Met O 20 Technical Note No. II/111. Available from UKMO, Bracknell U.K.

Speranza, A. (ed.), 1984: Special course on ALPEX results. Riv. di. Meteor Aero. XLIV.

Susskind, J. and E. Kalnay, 1985: Remote sensing of atmospheric parameters from HIRS2/MSU on TIROS-N. Proc First National Workshop on the Global Weather Experiment, Vol. II(i) pp. 361383 National Academy Press.

Tadjbakhsh, I. G., 1969: Utilization of timedependent data in a running solution of initial value problems. J. Appl. Meteor., 8, 389-391.

Talagrand, O., 1985: The adjoint model technique and meteorological applications. pp. 277-298 ECMWF Workshop on High Resolution Analysis.

Temperton, C. and D. L. Williamson, 1981: Normal mode initialisation for a multilevel grid-point model Part I: Linear Aspects. Mon. Wea. Rev., 109, 729-743.

Thiebaux, H. J., 1977: Extending estimation accuracy with anisotropic interpolation. Mon. Wea. Rev., 105, 691-699.

- , D.W. Shantz and H.L. Mitchell, 1986 : An algorithm for updating forecast initialisation procedures with periodic adjustment of the objective analysis scheme. Mon. Wea. Rev., 114, 1048-1066.

Tiedtke, M., J.F. Geleyn, A. Hollingsworth and J.F. Louis, 1979: ECMWF model parameterisation of sub grid scale processes. ECMWF Tech. Rept. 10.

- and J. Slingo, 1985: Development of the operational parameterisation scheme. ECMWF Tech. Memo. 108, available from ECMWF, Reading U.K.

- W. A. Heckley, J. Slingo, 1987: Tropical forecasts at ECMWF : Impact of revised physics and increased horizontal resolution. To appear in Quart. J. Roy. Meteor. Soc.
Tribbia, J. J., 1982: On variational normal mode initialisation. Mon. Wea. Rev., 110, 455-470.

Unden, P., 1983: A Method of correcting tidal biases in the data assimilation system. ECMWF Tech. Memo. 82, available from ECMWF, Reading U.K.

$\longrightarrow, 1984$ : Evaluation of analysis increments at model levels. ECMWF Tech. Memo. 94, available from ECMWF, Reading U.K.

Uppala, S., A. Hollingsworth, S. Tibaldi and P. Kallberg, 1984: Results from two recent observing system experiments at ECMWF. Proc. ECMWF Seminar on 'Data Assimilation Systems and Observing System Experiments with Particular Emphasis on FGGE', pp. 165-202; available from ECMWF.

Wahba, G. and J. Wendelberger, 1980: Some new matematical methods for variational objective analysis using splines and cross validation. Mon. Wea. Rev., 108, 1122-1143.

Wergen, W., 1982: Initialisation. In Proceedings of ECMWF Workshop on 'Current Problems in Data Assimilation', 31-58. Available from ECMWF Reading U.K.

- 1986: Diabatic non-linear normal mode initialisation for a spectral model with a hybrid vertical coordinate. ECMWF Tech. Rept. 59. Available from ECMWF, Reading, U.K. - and A. J. Simmons, 1986: Experiments with increased vertical resolution in the ECMWF model. to appear in Research Activities in Atmospheric and Oceanic Modelling, WMO, Geneva.

Williamson, D. L. and C. Temperton, 1981: Normal mode initialisation for a multi-level grid-point model, Part II : Nonlinear aspects. Mon. Wea. Rev., 109, 744-757.

and R. Daley, 1983: A unified analysisinitialization technique. Mon. Wea. Rev., 111, 1517-1536.

WMO-ICSU, 1985: Report of the Seminar on Progress in Diagnostic Studies of the Global Atmospheric Circulation as a result of the Global Weather Experiment. GARP Special Report 42. available from WMO, Casa postale 5, Geneva.

WMO, 1986a: Proc. ALPEX Conference, GARP Report 27. available from WMO, Geneva.

, 1986b : Preliminary assessment of ECMWF products used in Malaysia. WMO Bull., 35, 237240.

Wunsch, C. and J.F. Minster, 1982: Methods for box models and ocean circulation tracers : Mathematical programming and non-linear inverse theory. J. Geophys Res., 87, 5647-5662.

Wylie, D. P., B. B. Hinton, M. R. Howland and R. J. Lord, 1985: The auto-correlation of wind observations. Mon. Wea. Rev., 113, 849-857. 


\section{Appendix \\ Filtering and interpolation in statistical interpolation}

As shown in Section 4 we may write the vector of analysed values at the observation points as

$$
\left[a\left(r_{i}\right)\right]=\boldsymbol{P} \cdot(\boldsymbol{P}+\boldsymbol{D})^{-1} \cdot\left[d\left(r_{i}\right)\right]
$$

so that Eq. 4.1 may be rewritten as

$$
a\left(r_{k}\right)=\left[p\left(r_{k}, r_{i}\right)\right]^{*}(\boldsymbol{P})^{-1} \cdot\left[a\left(r_{i}\right)\right]
$$

Thus the analysis calculation may be thought of as a linear filter (Eq. 4.2) of the observed values to produce the analysed values at the observation points, followed by an interpolation (Eq. 4.3) of the filtered observations to the analysis points.

\section{a) The filter}

The nature of the filter in Eq. 4.2 is most conveniently discussed in terms of the ratio of observation error to prediction error. Both $\boldsymbol{P}$ and $\boldsymbol{D}$ are symmetric and positive definite. $\boldsymbol{P}$ may then be expressed in the form

$$
\boldsymbol{P}=\boldsymbol{E}\{\lambda\} \boldsymbol{E}^{*}
$$

where $E$ is an orthogonal matrix whose rows or columns are the eigenvectors of $\boldsymbol{P},\{\}$ denotes a diagonal matrix and the $\lambda_{i}$ are the corresponding eigenvalues. Define $\boldsymbol{Q}$ by

$$
\boldsymbol{Q}=\boldsymbol{E}\left\{\lambda^{-1 / 2}\right\} .
$$

Since $\boldsymbol{D}$ is positive definite and $\boldsymbol{Q}$ is nonsingular it follows that $Q^{*} \boldsymbol{D Q}$ is symmetric and positive definite, and may be written in the form

$$
Q^{*} D Q=R\{\nu\} R^{*}
$$

where the eigenvalues $\nu_{i}$ are all positive and $\boldsymbol{R}$ is orthogonal. $\boldsymbol{P}$ and $\boldsymbol{D}$ may now be diagonalised simultaneously:

$$
\begin{aligned}
& * R Q^{*} D Q R=\{\nu\} \\
& R^{*} Q^{*} P Q R=\{1\} .
\end{aligned}
$$

With a little manipulation it follows that

$$
\left[a\left(r_{i}\right)\right]=\left(\boldsymbol{R}^{*} \boldsymbol{Q}^{*}\right)^{-1}\left\{\frac{1}{1+\nu}\right\}\left(\boldsymbol{R}^{*} \boldsymbol{Q}^{*}\right)\left[d\left(r_{i}\right)\right]
$$

This shows clearly the nature of the filtering. The data undergoes a non-singular (but, in general, a non-orthogonal) change of basis $R^{*} Q^{*}$, the $j$ th component of the resulting vector is damped by the amount $1 / 1+\nu_{j}$ and the change of basis is reversed.

If $P$ and $\boldsymbol{D}$ are commutative with eigenvalues $\left[\lambda_{i}\right],\left[\sigma_{i}\right]$, and a common set of eigenvectors $\boldsymbol{E}$, then $\boldsymbol{R}=\boldsymbol{I}$, the identity matrix, and $\nu_{i}=\sigma_{i} / \lambda_{i}$. It follows that

$$
\left[a\left(r_{i}\right)\right]=\boldsymbol{E}\left\{\frac{1}{1+\sigma / \lambda}\right\} \boldsymbol{E}^{*}\left[d\left(r_{i}\right)\right] .
$$

In the simplest case of uniform uncorrelated observational error, $\boldsymbol{D}=\boldsymbol{I} \sigma$ and then the interpretation of $\nu_{i}$ as a ratio of observational error in a given component to forecast error in that component is quite obvious, as the damping on the $i$ th component is

$$
\frac{1}{1+\frac{\sigma}{\lambda_{i}}}
$$

If the ratio of observational error to forecast error in a given component is small the response is close to 1 and such components of the Ob-Fg differences are well analysed; if the ratio of observational error to forecast error is large then such components of the Ob-Fg differences are heavily damped. This simple result suggests that in the general case, when $\boldsymbol{P}$ and $\boldsymbol{D}$ are non-commutative, it is also reasonable to interpret $\nu_{i}$ in Eq. A.1 as a ratio of observation error in a given component to prediction error in that component.

\section{b) Interpolation}

The interpretation of Eq. 4.3 as an interpolation follows immediately from a standard result. The function $f(x)$ which interpolates a set of $N$ data points $F_{n}$ with a set of $N$ functions $g_{n}(x)$ is given by

$$
f(x)=\sum_{1}^{N} c_{n} g_{n}(x)
$$

where the coefficients $c_{n}$ satisfy

$$
\left[c_{n}\right]=G^{-1}\left[F_{n}\right]
$$

and the matrix $G$ is defined by 


$$
G_{i j}=g_{i}\left(x_{j}\right)
$$

A familiar example of this result is the Lagrange interpolation formula, where a clever choice of the functions $g_{n}(x)$ ensures that $G=I$.

\section{c) Filtering and Interpolation}

To see the intimate connection between the filtering and the interpolation, consider the interpolated value $a\left(r_{k}\right)$ in Eq. 4.3 when the filtered data vector $\left[a\left(r_{i}\right)\right]$ corresponds to one of the eigenvectors $\boldsymbol{e}_{j}$ of $\boldsymbol{P}$, the prediction error correlation matrix. The function

$$
\mathcal{E}_{j}\left(r_{k}\right)=\left[p\left(r_{k}, r_{i}\right)\right]^{*} \boldsymbol{P}^{-1} \boldsymbol{e}_{j}
$$

then interpolates the eigenvector exactly and we may call it an 'eigenfunction' of the matrix, where " ' indicates that we use the term in a very special sense. The relation between the filtering and the interpolation is now very simple if the matrices $\boldsymbol{P}$ and $\boldsymbol{D}$ commute. This assumption includes many cases of practical interest such as the case $D=\sigma I$, where the observation errors are uniform, random, and independent. For then if the original vector $\left[d\left(r_{i}\right)\right]$ is expanded in eigenvectors of $\boldsymbol{P}$ as

$$
\left[d\left(r_{i}\right)\right]=\sum_{1}^{N} c_{j} \boldsymbol{e}_{j}
$$

then the analysed values at the observation points are given by

$$
\left[a\left(r_{i}\right)\right]=\sum_{1}^{N} c_{j} \frac{1}{1+\nu_{j}} \boldsymbol{e}_{j},
$$

where $\nu_{i}$ is the ratio of observational error to prediction error in the $i$ th component. The analysed value at the point $r_{k}$ is given by

$$
a\left(r_{k}\right)=\sum_{1}^{N} c_{j} \frac{1}{1+\nu_{j}} \mathcal{E}_{j}\left(r_{k}\right)
$$

where the function $\mathcal{E}_{j}$ interpolates the vector $\boldsymbol{e}_{j}$ according to Eq. 4.3. 